M. Takeuchi

Nagoya Math. J.

Vol. 59 (1975), 9-44

\title{
ON SYMMETRIC SIEGEL DOMAINS
}

\section{Dedicated to the memory of Taira Honda}

\author{
MASARU TAKEUCHI
}

\section{Introduction}

Let $V$ be a convex cone in a real vector space $X, F: Y \times Y \rightarrow X^{c}$ a $V$-positive hermitian map on a complex vector space $Y$, and

$$
D(V, F)=\left\{(z, u) \in X^{c} \times Y ; \mathscr{I}_{m} z-F(u, u) \in V\right\}
$$

the Siegel domain associated to $V$ and $F . \quad D(V, F)$ is said to be symmetric, if for each point $p \in D(V, F)$ there exists an involutive holomorphic automorphism $\sigma_{p}$ of $D(V, F)$ such that the fixed point set of $\sigma_{p}$ consists of only the point $p$. Satake [6] showed that the symmetric Siegel domain $D(V, F)$ is characterized by the following three conditions (i), (ii) and (iii).

(i) $V$ is a self-dual homogeneous convex cone in $X$.

Let $G(V)$ denote the group of linear automorphisms of $V$ and take a point $e \in V$. Then the Lie algebra $g(V)$ of $G(V)$ is a reductive algebraic Lie algebra in $\operatorname{gr}(X)$ and the stabilizer

$$
\mathfrak{f}(V)=\{A \in \mathfrak{g}(V) ; A e=0\}
$$

of $e$ is a maximal compact subalgebra of $g(V)$. Let

$$
\mathfrak{g}(V)=\mathfrak{f}(V)+\mathfrak{p}(V)
$$

be the associated Cartan decomposition of $\mathrm{g}(V)$. Then, for each $x \in X$ there exists a unique element $T(x) \in \mathfrak{p}(V)$ satisfying $T(x) e=x$. Choose an inner product (, ) on $X$ such that if ${ }^{t} A$ denotes the transpose of $A \in \operatorname{gr}(X)$ with respect to this inner product, the Cartan involution associated to (C) is given by $A \mapsto-{ }^{t} A$. We define a hermitian inner product $\langle$,$\rangle on Y$ by

$$
\langle u, v\rangle=(e, F(u, v)) \quad \text { for } u, v \in Y,
$$

Received December 10, 1974. 
denoting the $C$-linear extension to $X^{c}$ of (, ) by the same notation (, ). Let Herm $(Y)$ be the real subspace of the space End $(Y)$ of $C$-linear endomorphisms of $Y$, consisting of those endomorphisms which are selfadjoint with respect to this hermitian inner product $\langle$,$\rangle . Then the$ second condition is stated as

(ii) For each $x \in X$ there exists $R(x) \in \operatorname{Herm}(Y)$ such that

$$
F(R(x) u, v)+F(u, R(x) v)=T(x) F(u, v) \quad \text { for } u, v \in Y .
$$

The above $R(x)$ is uniquely determined for $x \in X$, and so we have an $R$-linear map $R: X \rightarrow \operatorname{Herm}(Y)$ by the correspondence $x \mapsto R(x)$. The $C$-linear extension of the map $R$ will be also denoted by $R: X^{c} \rightarrow$ End $(Y)$. Then the third condition is stated as

(iii) For each $u, v \in Y, x \in X$, we have

$$
R(F(u, v)) R(x) u=R(F(u, R(x) v)) u .
$$

In this note, we shall classify Siegel domains satisfying the conditions (i) and (ii), and then determine the ones with the condition (iii) among these Siegel domains. Our problem reduces to the classification in the case where $V$ is an indecomposable self-dual homogeneous convex cone, and so we shall restrict ourselves to consider $D(V, F)$ for such convex cone $V$.

Take a maximal abelian subalgebra $\mathfrak{a}$ in $\mathfrak{p}(V)$ and put $m=\operatorname{dim} \mathfrak{a}$. Then the multiplicities of a-roots of $\mathrm{g}(V)$ are the same for all a-roots. We define a positive integer $\nu$ to be this multiplicity if $m \geqq 2$, and $\nu=1$ if $m=1$. Then the pair $(m, \nu)$ is a complete invariant for the linear isomorphism classes of indecomposable self-dual homogeneous convex cones, and the possibilities of $(m, \nu)$ are $m=1, \nu=1 ; m=2, \nu \geqq 1 ; m$ $=3, \nu=1,2,4$ or $8 ; m \geqq 4, \nu=1,2$ or 4 . The cone with the invariant $(m, \nu)$ will be denoted by $V_{m}^{\nu}$. We associate to such $(m, \nu)$ an algebra $\boldsymbol{F}_{m}^{\nu}$ over $\boldsymbol{R}$ in the following way: $\boldsymbol{F}_{m}^{\nu}=$ the Clifford algebra $C_{\nu-1}$ associated to the negative definite quadratic form on $\boldsymbol{R}^{\nu-1}$, if $m=2 ; \boldsymbol{F}_{m}^{\nu}=$ the division algebra over $\boldsymbol{R}$ of dimension $\nu$, if $m \neq 2$. Let $\lambda \mapsto \bar{\lambda}$ denote the canonical involution of $\boldsymbol{F}_{m}^{\nu}$. We define a subspace $\boldsymbol{P}_{m}^{\nu}$ of $\boldsymbol{F}_{m}^{\nu}$ by $P_{m}^{\nu}=\boldsymbol{R} 1+\boldsymbol{R}^{\nu-1} \subset C_{\nu-1}$ if $m=2 ; P_{m}^{\nu}=\boldsymbol{F}_{m}^{\nu}$ if $m \neq 2$, and denote by $\mathscr{P}: \boldsymbol{F}_{m}^{\nu} \rightarrow P_{m}^{\nu}$ the orthogonal projection to $P_{m}^{\nu}$ relative to the standard inner product of $F_{m}^{\nu}$. Let $c_{0}=1, c_{1}, \cdots, c_{\nu-1}$ be the standard basis of $P_{m}^{v}$ with relations 


$$
c_{i}^{2}=-1(1 \leqq i \leqq \nu-1), c_{i} c_{k}+c_{k} c_{i}=0(1 \leqq i<k \leqq \nu-1) .
$$

We denote by $\operatorname{Aut}^{*}\left(\boldsymbol{F}_{m}^{\nu}, \mathscr{P}\right)$ the group of those automorphisms of the algebra $\boldsymbol{F}_{m}^{\nu}$ commuting with $\lambda \mapsto \bar{\lambda}$ and $\mathscr{P}$, and by $\mathfrak{M} *\left(\boldsymbol{F}_{m}^{\nu}\right)$ the set of unitary equivalence classes of $\boldsymbol{F}_{m}^{\nu}$-modules $W$ over $\boldsymbol{C}$ with a hermitian inner product $\langle$,$\rangle satisfying the condition$

$$
\langle\lambda u, v\rangle=\langle u, \bar{\lambda} v\rangle \quad \text { for } \lambda \in \boldsymbol{F}_{m}^{\nu}, u, v \in W .
$$

We define $\mathfrak{M}(m, \nu)$ to be the orbit space $\operatorname{Aut}^{*}\left(\boldsymbol{F}_{m}^{\nu}, \mathscr{P}\right) \backslash \mathfrak{M} *\left(\boldsymbol{F}_{m}^{\nu}\right)$ relative to the natural action of $\operatorname{Aut}^{*}\left(\boldsymbol{F}_{m}^{\nu}, \mathscr{P}\right)$ on $\mathfrak{M}^{*}\left(\boldsymbol{F}_{m}^{\nu}\right)$. Then our results are stated as follows.

THEOREM A. The set $\mathfrak{M}(m, \nu)$ is in a bijective correspondence with the set of affine isomorphism classes of those Siegel domains $D(V, F)$ satisfying $V=V_{m}^{\nu}$ and the condition (ii).

THEOREM B. ${ }^{1)}$ Let $W$ be an $\boldsymbol{F}_{m}^{\nu}$-module with a hermitian inner product $\langle$,$\rangle satisfying the condition (*)$, and $D\left(V_{m}^{\nu}, F\right)$ the Siegel domain corresponding to $W$. Then $D\left(V_{m}^{\nu}, F\right)$ satisfies the condition (iii) if and only if $m=1$, or $m \geqq 2$ and

$$
\sum_{i=0}^{\nu-1}\left\langle c_{i} u, v\right\rangle\left\langle c_{i} u, w\right\rangle=0 \quad \text { for } u, v, w \in W .
$$

By means of Theorems $A$ and $B$, we get a new classification of symmetric bounded domains without the use of the classification of semisimple Lie algebras of hermitian type.

Finally we note (cf. Tsuji [8]) that if an $F_{m}^{\nu}$-module $W(m \geqq 2)$ does not satisfy the condition (\#), then the corresponding Siegel domain $D\left(V_{m}^{\nu}, F\right)$ has no infinitesimal automorphisms other than infinitesimal affine automorphisms.

\section{Homogeneous Siegel domains}

In this section we recall definitions and fundamental results on homogeneous Siegel domains. For homogeneous cones and T-algebras, see [9], [10], [11].

Let $X$ be a finite dimensional real vector space. An open subset $V$ of $X$ is called a convex cone, if (1) $x, y \in V, 0<\lambda<1 \Rightarrow \lambda x+(1-\lambda) y$ $\in V$, (2) $x \in V, \lambda>0 \Rightarrow \lambda x \in V$, and (3) $V$ does not contain any straight

1) In the case of $F_{2}^{\nu}$, the same result is obtained by T. Tsuji (unpublished). 
line. We denote by $G(V)$ the subgroup of $G L(X)$ consisting of those $\varphi \in G L(X)$ satisfying $\varphi V=V . \quad V$ is said to be homogeneous if $G(V)$ acts transitively on $V$. Two convex cones $V \subset X$ and $V^{\prime} \subset X^{\prime}$ are said to be linearly isomorphic, if there exists a linear isomorphism $\varphi: X \rightarrow X^{\prime}$ satisfying $\varphi V=V^{\prime}$. Let $V_{i} \subset X_{i}(1 \leqq i \leqq r)$ be convex cones. Put $X$ $=X_{1}+\cdots+X_{r}$ (direct sum) and $V=V_{1}+\cdots+V_{r}$. Then $V$ is a convex cone in $X$. This cone is called the direct product of the $V_{i}$ $(1 \leqq i \leqq r)$ and denoted by $V=V_{1} \times \cdots \times V_{r}$. A convex cone $V$ is said to be decomposable if it is linearly isomorphic to the direct product $V_{1} \times V_{2}$ of non-trivial convex cones $V_{1}$ and $V_{2}$; otherwise it is said to be indecomposable. For a convex cone $V \subset X$, the subset $V^{*}$ of the dual space $X^{*}$ of $X$ defined by

$$
V^{*}=\left\{\xi \in X^{*} ; \xi(x)>0 \text { for any } x \in \bar{V}-\{0\}\right\},
$$

is also a convex cone. $V$ is said to be self-dual if there exists an inner product (, ) on $X$ such that the canonical isomorphism $\varphi: X \rightarrow X^{*}$ defined by $\varphi(x)(y)=(x, y)$ satisfies $\varphi V=V^{*}$. Each (self-dual) homogeneous convex cone is linearly isomorphic to the direct product $V_{1} \times \cdots \times V_{r}$ of indecomposable (self-dual) homogeneous convex cones $V_{i}(1 \leqq i \leqq r)$, where the $V_{i}$ are unique up to linear isomorphism and permutation (Vinberg [9], [10]).

Let $V \subset X$ be a convex cone and $Y$ a finite dimensional complex vector space. A map $F: Y \times Y \rightarrow X^{c}$, where $X^{c}$ denotes the complexification of $X$, is called a hermitian map, if (1) $F\left(\lambda_{1} u_{1}+\lambda_{2} u_{2}, v\right)=\lambda_{1} F\left(u_{1}, v\right)$ $+\lambda_{2} F\left(u_{2}, v\right)$ for $\lambda_{1}, \lambda_{2} \in C, u_{1}, u_{2}, v \in Y$, and (2) $\overline{F(u, v)}=F(v, u)$ for $u, v \in Y$. It is said to be $V$-positive, if (1) $F(u, u)$ belongs to the closure $\bar{V}$ of $V$ for each $u \in Y$, and (2) $F(u, u)=0 \Rightarrow u=0$. For a $V$-positive hermitian map $F$, the domain $D(V, F)$ in $X^{c} \times Y$ defined by

$$
D(V, F)=\left\{(z, u) \in X^{c} \times Y ; \mathscr{I}_{m} z-F(u, u) \in V\right\},
$$

is called the Siegel domain associated to $V$ and $F$. It is known (PjateckiiSapiro [5]) that $D(V, F)$ is holomorphically isomorphic to a bounded domain in $X^{c} \times Y$. We denote by $A(D(V, F))$ the group of those affine automorphisms $\varphi$ of $X^{c} \times Y$ satisfying $\varphi(D(V, F))=D(V, F) . \quad D(V, F)$ is said to be affinely homogeneous if $A(D(V, F)$ ) acts transitively on $D(V, F)$. A Siegel domain satisfying the Satake's conditions (i) and (ii) is always affinely homogeneous (cf. $\S 3$ ). If $D(V, F)$ is affinely homogeneous, then $V$ is homogeneous (Pjateckii-Šapiro [5]). Two Siegel 
domains $D(V, F) \subset X^{c} \times Y$ and $D\left(V^{\prime}, F^{\prime}\right) \subset X^{\prime c} \times Y^{\prime}$ are said to be affinely isomorphic if there exists an affine isomorphism $\varphi: X^{c} \times Y \rightarrow$ $X^{\prime C} \times Y^{\prime}$ satisfying $\varphi(D(V, F))=D\left(V^{\prime}, F^{\prime}\right)$. Let $D\left(V_{i}, F_{i}\right) \subset X_{i}^{\boldsymbol{c}} \times Y_{i}(1$ $\leqq i \leqq r)$ be Siegel domains. Put $V=V_{1} \times \cdots \times V_{r} \subset X=X_{1}+\cdots+X_{r}$ and $Y=Y_{1}+\cdots+Y_{r}$. We define a $V$-positive hermitian map $F: Y \times Y$ $\rightarrow X^{C}$ by

$$
F\left(\sum_{i} u_{i}, \sum_{i} v_{i}\right)=\sum_{i} F_{i}\left(u_{i}, v_{i}\right) \quad \text { for } u_{i}, v_{i} \in Y_{i}(1 \leqq i \leqq r)
$$

The Siegel domain $D(V, F)$ associated to $V$ and $F$ is called the direct product of the $D\left(V_{i}, F_{i}\right)(1 \leqq i \leqq r)$ and denoted by $D(V, F)=D\left(V_{1}, F_{1}\right)$ $\times \cdots \times D\left(V_{r}, F_{r}\right)$. A Siegel domain $D(V, F)$ is said to be decomposable if it is affinely isomorphic to the direct product $D\left(V_{1}, V_{1}\right) \times D\left(V_{2}, F_{2}\right)$ of non-trivial Siegel domains $D\left(V_{1}, F_{1}\right)$ and $D\left(V_{2}, F_{2}\right)$; otherwise it is said to be indecomposable.

In general, a complex manifold $D$ is said to be homogeneous if the group of holomorphic automorphisms of $D$ acts transitively on $D$. $D$ is said to be symmetric, if for each $p \in D$ there exists an involutive holomorphic automorphism $\sigma_{p}$ of $D$ such that the fixed point set of $\sigma_{p}$ consists of only the point $p$. It is known (Vinberg-Gindikin-Pjateckii-Šapiro [12]) that the set of affine isomorphism classes of affinely homogeneous Siegel domains is in the bijective correspondence with the set of holomorphic isomorphism classes of homogeneous bounded domains. Each symmetric Siegel domain is affinely homogeneous, since the Bergman metric of a Siegel domain is complete (Nakajima [3]), and a homogeneous Siegel domain is affinely homogeneous (Kaup-Matsushima-Ochiai [2]). Thus we have the bijective correspondence between the set of affine isomorphism classes of symmetric Siegel domains and the set of holomorphic isomorphism classes of symmetric homogeneous bounded domains. Furthermore it is known (Kaneyuki [1]) that each affinely homogeneous Siegel domain $D(V, F)$ is affinely isomorphic to the direct product $D\left(V_{1}, F_{1}\right) \times \cdots \times D\left(V_{r}, F_{r}\right)$ of indecomposable affinely homogeneous Siegel domains $D\left(V_{i}, F_{i}\right)(1 \leqq i \leqq r)$, where the $D\left(V_{i}, F_{i}\right)$ are unique up to affine isomorphism and permutation, and that $D(V, F)$ is indecomposable if and only if $V$ is indecomposable. Thus our problem reduces to the case where $V$ is indecomposable.

Now we recall the notion of $T$-algebras. Let 


$$
A=\sum_{1 \leqq i, k \leqq m} A_{i k}
$$

be a finite dimensional bigraded distributive algebra over $\boldsymbol{R}$ and $a \mapsto a^{*}$ be an involutive anti-automorphism of $A . A$ is called a matrix algebra with involution of rank $m$, if (1) $A_{i k} A_{k \ell} \subset A_{i \ell}$, and $A_{i k} A_{p q}=\{0\}$ if $k \neq p$, (2) $A_{i k}^{*}=A_{k i}$, and (3) $A_{i i}$ is a subalgebra of $A$ with the unit element $e_{i}$, for each $i$. An element

$$
a=\sum_{1 \leqq i, k \leqq m} a_{i k}, \quad a_{i k} \in A_{i k}
$$

of $A$ will be often denoted by the matricial form $a=\left(a_{i k}\right)$. The general element of $A_{i k}$ (resp. of $A$ ) will be denoted by $a_{i k}, b_{i k}, c_{i k}, \cdots$ (resp. by $a, b, c, \cdots)$. A matrix algebra $A$ with involution of rank $m$ is called a T-algebra of rank $m$, if

( I ) There exists an algebra isomorphism $\theta: A_{i i} \rightarrow \boldsymbol{R}$ for each $i$.

( II ) $e_{i} a_{i k}=a_{i k}$.

(III) There exist $n_{i}>0(1 \leqq i \leqq m)$ such that

$$
n_{i} \theta\left(a_{i k} b_{k i}\right)=n_{k} \theta\left(b_{k i} a_{i k}\right) \text {. }
$$

(IV) $\theta\left(a_{i k} a_{i k}^{*}\right)>0$ if $a_{i k} \neq 0$.

$(\mathrm{V}) \quad a_{i k}\left(b_{k \ell} c_{\ell i}\right)=\left(a_{i k} b_{k \ell}\right) c_{\ell i}$.

(VI) $\quad a_{i k}\left(b_{k \ell} c_{\ell p}\right)=\left(a_{i k} b_{k \ell}\right) c_{\ell p}$ if $i<k<\ell$ and $k<p$.

(VII) $a_{i k}\left(b_{k \ell} b_{k \ell}^{*}\right)=\left(a_{i k} b_{k \ell}\right) b_{k \ell}^{*}$ if $i<k<\ell$.

For a $T$-algebra $A$ of rank $m$, we define

$$
\begin{aligned}
n_{i k} & =\operatorname{dim} A_{i k} & & \text { for } 1 \leqq i, k \leqq m, \\
\operatorname{tr} a & =\sum_{1 \leqq i \leqq m} n_{i} \theta\left(a_{i i}\right) & & \text { for } a=\left(a_{i k}\right) \in A, \\
(a, b) & =\operatorname{tr} a b^{*} & & \text { for } a, b \in A .
\end{aligned}
$$

Then (, ) is an inner product on $A$ satisfying

$$
\begin{gathered}
\left(a^{*}, b^{*}\right)=(a, b), \\
(a b, c)=\left(b, a^{*} c\right)=\left(a, c b^{*}\right) .
\end{gathered}
$$

It follows from (II) that $e=\sum_{1 \leqq i \leqq m} e_{i}$ is the unit element of the algebra A. A permutation $i \mapsto \tilde{i}$ of the set $\{1,2, \cdots, m\}$ of indices is said to be admissible for $A$, if $i<k, \tilde{i}>\tilde{k}$ imply $n_{i k}=0$. The renaming $A_{i k}$ $\sim A_{\tilde{i} \tilde{k}}$ of the grading of $A$ by an admissible permutation $i \mapsto \tilde{i}$, is called an inessential change of the grading of $A$. T-algebras $A$ of rank 
$m$ and $A^{\prime}$ of rank $m^{\prime}$ are said to be isomorphic, if $m=m^{\prime}$ and there exists an algebra isomorphism of $A$ onto $A^{\prime}$ such that it becomes an isomorphism as bigraded involutive algebras (i.e., it preserves gradings and commutes with involutions), after an inessential change of the grading of $A$.

For a $T$-algebra $A$ of rank $m$, we put

$$
\begin{aligned}
& X(A)=\left\{a \in A ; a^{*}=a\right\}, \\
& \mathscr{T}(A)=\left\{\left(a_{i k}\right) \in A ; a_{i k}=0(i>k), \theta\left(a_{i i}\right)>0(1 \leqq i \leqq m)\right\}, \\
& V(A)=\left\{t t^{*} ; t \in \mathscr{T}(A)\right\} \subset X(A) .
\end{aligned}
$$

Then (Vinberg [10]) $V(A)$ is a homogeneous convex cone in $X(A)$ and the correspondence $A \backsim V(A)$ induces a bijection from the set of isomorphism classes of $T$-algebras onto the set of linear isomorphism classes of homogeneous convex cones. Furthermore it is known (Vinberg [11]) that $V(A)$ is an indecomposable self-dual convex cone if and only if $m=1$, or $m \geqq 2$ and all $n_{i k}(i \neq k)$ are the same non-zero integer. For such $T$-algebra, we put $\nu=1$ if $m=1$, and $\nu=n_{i k}(i \neq k)$ if $m \geqq 2$. Then the pair $(m, \nu)$ serves as a complete invariant for the linear isomorphism classes of indecomposable self-dual homogeneous convex cones. It is not difficult to see that our invariant $(m, \nu)$ coincides with the one given in Introduction. We know that the possibilities of $(m, \nu)$ are as those in Introduction, seeing the classification of Vinberg [9] of such convex cones. For such pair $(m, \nu)$ we associate an algebra $\boldsymbol{F}_{m}^{\nu}$ over $\boldsymbol{R}$ as in Introduction. Then the $T$-algebra $A_{m}^{\nu}$ with the invariant $(m, \nu)$ is constructed explicitly in the following way.

Let first $m=2$, and

$$
C_{\nu-1}=\sum_{0 \leqq s \leqq \nu-1}\left(C_{\nu-1}\right)_{s}, \quad\left(C_{\nu-1}\right)_{s}=\sum_{i_{1}<\cdots<i_{s}} R c_{i_{1}} \cdots c_{i_{s}}
$$

be the usual graduation of $C_{\nu-1}=\boldsymbol{F}_{m}^{\nu}$. Identifying $\boldsymbol{R}$ with $\left(C_{\nu-1}\right)_{0}=\boldsymbol{R} 1$, we define a linear map $\mathscr{R}_{e}: \boldsymbol{F}_{m}^{\nu} \rightarrow \boldsymbol{R}$ by the projection $C_{\nu-1} \rightarrow\left(C_{\nu-1}\right)_{0}$. Putting $P_{m}^{\nu}=\left(C_{\nu-1}\right)_{0}+\left(C_{\nu-1}\right)_{1}$, we also define a linear map $\mathscr{P}: \boldsymbol{F}_{m}^{\nu} \rightarrow P_{m}^{\nu}$ by the projection $C_{\nu-1} \rightarrow\left(C_{\nu-1}\right)_{0}+\left(C_{\nu-1}\right)_{1}$. Denoting by $\lambda \mapsto \bar{\lambda}$ the canonical positive involution of $C_{\nu-1}$ (defined by the correspondence $c_{i_{1}} c_{i_{2}} \cdots c_{i_{s}} \mapsto$ $\left(-c_{i_{s}}\right) \cdots\left(-c_{i_{2}}\right)\left(-c_{i_{1}}\right)$, we define the standard inner product $($,$) on F_{m}^{\nu}$ by

$$
(\lambda, \mu)=\mathscr{R} e \lambda \bar{\mu} \quad \text { for } \lambda, \mu \in \boldsymbol{F}_{m}^{\nu}
$$


Note that $P_{m}^{\nu}$ is invariant under the involution $\lambda \mapsto \bar{\lambda}$ and

$$
\mathscr{R} e \lambda=\frac{1}{2}(\mathscr{P} \lambda+\overline{\mathscr{P} \lambda}) \quad \text { for } \lambda \in F_{m}^{\nu} \text {. }
$$

Let next $m \neq 2$. Then $\boldsymbol{F}_{m}^{\nu}$ is the division algebra over $\boldsymbol{R}$ of dimension $\nu$, and so we have the direct sum decomposition

$$
\boldsymbol{F}_{m}^{\nu}=\boldsymbol{R} 1+\left\{\lambda \in \boldsymbol{F}_{m}^{\nu} ; \bar{\lambda}=-\lambda\right\},
$$

where $\lambda \mapsto \bar{\lambda}$ denotes the canonical involution of $\boldsymbol{F}_{m}^{\nu}$. Identifying $\boldsymbol{R}$ with $\boldsymbol{R} 1$ and putting $P_{m}^{\nu}=F_{m}^{\nu}$, we define linear maps $\mathscr{R}_{e}: F_{m}^{\nu} \rightarrow \boldsymbol{R}$ and $\mathscr{P}: \boldsymbol{F}_{m}^{\nu}$ $\rightarrow P_{m}^{\nu}$ by the projection $\boldsymbol{F}_{m}^{\nu} \rightarrow \boldsymbol{R} 1$ and the identity map of $\boldsymbol{F}_{m}^{\nu}$ respectively. Then (1.2) holds also for these $\boldsymbol{F}_{m}^{\nu}$. The standard inner product (, ) on $F_{m}^{\nu}$ is also defined by the formula (1.1). Note that then for each $m$ the involution $\lambda \mapsto \bar{\lambda}$ commutes with $\mathscr{R} e$ and $\mathscr{P}$.

Now, for a general pair $(m, \nu)$, we denote by $\hat{A}_{m}^{v}$ the algebra $M_{m}\left(\boldsymbol{F}_{m}^{\nu}\right)$ of all matrices of degree $m$ with coefficients in $\boldsymbol{F}_{m}^{\nu}$. The product in $\hat{A}_{m}^{v}$ will be denoted by $a \cdot b$. Put $a^{*}={ }^{t} \bar{a}$ for $a \in \hat{A}_{m}^{v}$. Then $\hat{A}_{m}^{v}$ becomes a matrix algebra with involution of rank $m$. A linear endomorphism $p$ of $\hat{A}_{m}^{v}$ is defined by

$$
(p a)_{i k}= \begin{cases}\mathscr{P} a_{i k} & \text { if } i \neq k \\ \mathscr{R} e a_{i k} & \text { if } i=k .\end{cases}
$$

We put $A_{m}^{\nu}=p\left(\hat{A}_{m}^{\nu}\right)$ and define a product in $A_{m}^{\nu}$ by $a b=p(a \cdot b)$ for $a, b \in A_{m}^{\nu} . \quad A_{m}^{\nu}$ has the natural bigrading induced from the one of $\hat{A}_{m}^{v}$. The involution $a \mapsto a^{*}$ of $\hat{A}_{m}^{v}$ leaves $A_{m}^{v}$ invariant and induces an involution of $A_{m}^{\nu}$. Relative to these grading and involution, $A_{m}^{\nu}$ becomes a $T$ algebra of rank $m$, by taking $n_{i}=1$ for each $i$. This $T$-algebra $A_{m}^{\nu}$ is the required one.

Put

$$
X_{m}^{\nu}=X\left(A_{m}^{\nu}\right), \quad V_{m}^{\nu}=V\left(A_{m}^{\nu}\right) .
$$

Then $X_{m}^{\nu}$ coincides with the space

$$
H_{m}\left(P_{m}^{\nu}\right)=\left\{a \in M_{m}\left(P_{m}^{\nu}\right) ; t \bar{a}=a\right\}
$$

of all hermitian matrices in the space $M_{m}\left(P_{m}^{\nu}\right)$ of matrices of degree $m$ with coefficients in $P_{m}^{v}$. It becomes a compact simple Jordan algebra by the product

$$
x \square y=\frac{1}{2}(x y+y x) \quad \text { for } x, y \in X_{m}^{\nu} .
$$


The unit element $e$ of the $T$-algebra $A_{m}^{\nu}$ is also the unit element of the Jordan algebra $X_{m}^{\nu}$. The cone $V_{m}^{\nu}$ is also represented as the interior of the subset $\left\{x \square x ; x \in X_{m}^{\nu}\right\}$ of $X_{m}^{\nu}$. We define the standard inner product $\left(\right.$, ) on $X_{m}^{\nu}$ by

$$
(x, y)=\operatorname{Tr} x \square y=\mathscr{R} e(\operatorname{Tr} x \cdot y) \quad \text { for } x, y \in X_{m}^{\nu},
$$

where $\operatorname{Tr}$ denotes the usual trace of a matrix. This coincides with the restriction to $X_{m}^{\nu}$ of the inner product tr $a b^{*}$ of the $T$-algebra $A_{m}^{\nu}$. The cone $V_{m}^{\nu}$ is self-dual with respect to this inner product. We define an injective linear map $\psi: X_{m}^{\nu} \rightarrow \operatorname{gl}\left(X_{m}^{\nu}\right)$ by

$$
\psi(x) y=x y+y x=2 x \square y \quad \text { for } x, y \in X_{m}^{\nu} .
$$

Let $\mathfrak{p}_{m}^{\nu}=\psi\left(X_{m}^{\nu}\right)$ and $\mathfrak{f}_{m}^{\nu}$ be the subalgebra of $\mathfrak{g l}\left(X_{m}^{\nu}\right)$ consisting of all derivations of the Jordan algebra $X_{m}^{\nu}$. Then the Lie algebra $g\left(V_{m}^{\nu}\right)$ of the group $G\left(V_{m}^{\nu}\right)$ is given by

$$
\mathrm{g}\left(V_{m}^{\nu}\right)=\mathfrak{H}_{m}^{\nu}+\mathfrak{p}_{m}^{\nu},
$$

and $\mathfrak{f}_{m}^{\nu}$ coincides with the stabilizer of the point $e \in V_{m}^{\nu}$ in $\mathfrak{g}\left(V_{m}^{\nu}\right)$. Furthermore the above is a Cartan decomposition of $g\left(V_{m}^{\nu}\right)$ and the associate Cartan involution is given by $A \mapsto-{ }^{t} A$ with respect to the standard inner product of $X_{m}^{\nu}$. If we put

$$
T(x)=\frac{1}{2} \psi(x) \quad \text { for } x \in X_{m}^{\nu},
$$

we have $T(x) \in \mathfrak{p}_{m}^{\nu}$ and $T(x) e=x$ for each $x \in X_{m}^{\nu}$.

Next we recall the notion of $S$-algebras. Let

$$
C=\sum_{1 \leqq i,} C_{k \leqq m+1} C_{i k}
$$

be a $T$-algebra of rank $m+1$ and $j$ complex structures on $C_{i, m+1}, C_{m+1, i}$ $(1 \leqq i \leqq m) . \quad C$ is called an S-algebra of rank $m$, if it satisfies the following additional conditions:

(VIII) $\quad a_{i k}\left(j b_{k, m+1}\right)=j\left(a_{i k} b_{k, m+1}\right) \quad$ if $1 \leqq i<k \leqq m$.

( IX ) $j\left(a_{i, m+1}^{*}\right)=\left(j a_{i, m+1}\right)^{*} \quad$ for each $1 \leqq i \leqq m$.

( X ) $\theta\left(j a_{i, m+1}\left(j a_{i, m+1}\right)^{*}\right)=\theta\left(a_{i, m+1} a_{i, m+1}^{*}\right) \quad$ for each $1 \leqq i \leqq m$.

$j$ will be called the partial complex structure of $C$. For an $S$-algebra $C$ of rank $m$, we put 


$$
\begin{aligned}
A & =\sum_{1 \leqq i, k \leqq m} C_{i k}, \\
B & =\sum_{1 \leqq i \leqq m}\left(C_{i, m+1}+C_{m+1, i}\right), \\
X & =\left\{a \in A ; a^{*}=a\right\}, \\
Y & =\left\{a \in B ; a^{*}=a\right\} .
\end{aligned}
$$

Then $A$ becomes a $T$-algebra of rank $m$ and is called the T-algebra associated to $C$. The complex structure $j$ on $B$ leaves $Y$ invariant and so induces a complex structure $j$ on $Y$. This $j$ makes $Y$ into a complex vector space in the natural way, which will be denoted by $(Y, j)$. The inner product (, ) on $C$ defined by the $T$-algebra structure on $C$ satisfies

$$
(j a, j b)=(a, b) \quad \text { for } a, b \in B .
$$

Remark. It is known (Takeuchi [7]) that the positive numbers

$$
n_{i}^{0}=2\left(2+\sum_{\substack{1 \leqq k \leqq m \\ k \neq i}} n_{i k}+\frac{1}{2} n_{i, m+1}\right) \quad(1 \leqq i \leqq m), \quad n_{m+1}^{0}=1
$$

satisfy the axiom (III).

A permutation $i \mapsto \tilde{i}$ of the set $\{1,2, \cdots, m\}$ is said to be admissible for $C$, if $i<k, \tilde{i}>\tilde{k}$ imply $n_{i k}=0$. The renaming $C_{i k} \sim C_{\tilde{i} \tilde{k}}$ $(1 \leqq i, k \leqq m), \quad C_{i, m+1} \backsim C_{\tilde{i}, m+1}, \quad C_{m+1, i} \backsim C_{m+1, i}(1 \leqq i \leqq m)$ of the grading of $C$ by an admissible permutation $i \mapsto \tilde{i}$, is called an inessential change of the grading of $C$. S-algebras $C$ of rank $m$ and $C^{\prime}$ of rank $m^{\prime}$ are said to be isomorphic, if $m=m^{\prime}$ and there exists an algebra isomorphism of $C$ onto $C^{\prime}$ such that it becomes an isomorphism as a bigraded involutive algebra with partial complex structure (i.e., it preserves gradings and commutes with involutions and partial complex structures), after an inessential change of the grading of $C$.

Let $C$ be an $S$-algebra of rank $m$. Put

$$
\hat{u}=\sum_{1 \leqq i \leqq m} u_{i, m+1}, \quad \underline{u}=\sum_{1 \leqq i \leqq m} u_{m+1, i} \quad \text { for } u=\left(u_{i k}\right) \in Y,
$$

and define a symmetric bilinear map $\Phi: Y \times Y \rightarrow X$ by

$$
\Phi(u, v)=\frac{1}{2}(\hat{u} v+\hat{v} u) \quad \text { for } u, v \in Y .
$$

Then it satisfies $\Phi(j u, j v)=\Phi(u, v)$ for $u, v \in Y$, and hence the map $F:(Y, j) \times(Y, j) \rightarrow X^{c}$ defined by

$$
F(u, v)=\frac{1}{2}\{\Phi(u, v)+\sqrt{-1} \Phi(u, j v)\} \quad \text { for } u, v \in Y
$$


is a hermitian map. The maps $\Phi$ and $F$ are said to be associated to $C$. It is known (Takeuchi [7]) that $F$ is a $V(A)$-positive hermitian map. The Siegel domain $D(V(A), F) \subset X^{c} \times(Y, j)$ associated to $V(A)$ and $F$ will be denoted by $D(C)$. Then (Takeuchi [7]) the correspondence $C \backsim D(C)$ induces a bijection from the set of isomorphism classes of $S$-algebras onto the set of affine isomorphism classes of affinely homogeneous Siegel domains.

\section{Linear automorphisms of a Siegel domain}

We consider the pair $\mathscr{S}=\left(S ;\left\{m_{\alpha}\right\}_{1 \leqq \alpha \leqq \mu+1}\right)$ of a matrix algebra

$$
S=\sum_{1 \leqq \alpha, \beta \leqq \mu+1} S_{\alpha \beta}
$$

with involution $u \mapsto u^{*}$ of $\operatorname{rank} \mu+1$ and the set $\left\{m_{\alpha}\right\}_{1 \leqq \alpha \leqq \mu+1}$ of $\mu+1$ positive integers with $m_{\mu+1}=1$, such that (1) partial complex structures $j$ on $S_{\alpha, \mu+1}, S_{\mu+1, \alpha}(1 \leqq \alpha \leqq \mu)$ are defined; (2) linear endomorphisms $\mathscr{P}$ on $S_{\alpha \alpha}(1 \leqq \alpha \leqq \mu+1)$ are defined; (3) for each $\alpha(1 \leqq \alpha \leqq \mu+1)$, there exists an algebra isomorphism $\vartheta: S_{\alpha \alpha} \rightarrow F_{m_{\alpha}}^{\nu_{\alpha}}$ for some $\nu_{\alpha}$, satisfying $\overline{\vartheta \lambda}$ $=\vartheta\left(\lambda^{*}\right), \mathscr{P}(\vartheta \lambda)=\vartheta(\mathscr{P} \lambda)$ for $\lambda \in S_{\alpha \alpha}$. Such pair $\mathscr{S}$ is called an $S$-system of rank $\mu$. The general element of $S_{\alpha \beta}$ (resp. of $S$ ) will be denoted by $u_{\alpha \beta}, v_{\alpha \beta}, w_{\alpha \beta}, \cdots$ (resp. by $\left.u, v, w, \cdots\right)$.

Let $\mathscr{S}=\left(S ;\left\{m_{\alpha}\right\}_{1 \leqq \alpha \leqq \mu+1}\right)$ be an $S$-system of rank $\mu$ and $\varepsilon_{\alpha}$ the unit element of the algebra $S_{\alpha \alpha}(1 \leqq \alpha \leqq \mu+1)$. Identifying $\boldsymbol{R}$ with $\boldsymbol{R}_{\alpha}$, we define a linear map $\mathscr{R}_{e}: S_{\alpha \alpha} \rightarrow R$ by

$$
\mathscr{R} e \lambda=\frac{1}{2}\left(\mathscr{P} \lambda+(\mathscr{P} \lambda)^{*}\right) \quad \text { for } \lambda \in S_{\alpha \alpha} \text {. }
$$

Then we have

$$
\mathscr{R} e \lambda=\mathscr{R} e(\vartheta \lambda) \quad \text { for } \lambda \in S_{\alpha \alpha}
$$

and the involution $u \mapsto u^{*}$ of $S$ commutes with $\mathscr{P}$ and $\mathscr{R} e$ on each $S_{\alpha \alpha}$. A permutation $\alpha \mapsto \tilde{\alpha}$ of the set $\{1,2, \cdots, \mu\}$ of indices is said to be $a d$ missible for $\mathscr{S}$, if $\alpha<\beta$, $\tilde{\alpha}>\tilde{\beta}$ imply $S_{\alpha \beta}=\{0\}$. The renaming $S_{\alpha \beta}$ $\leadsto S_{\tilde{\alpha} \tilde{\beta}}(1 \leqq \alpha, \beta \leqq \mu), S_{\alpha, \mu+1} \sim S_{\tilde{\alpha}, \mu+1}, S_{\mu+1, \alpha} \sim S_{\mu+1, \tilde{\alpha}}(1 \leqq \alpha \leqq \mu), m_{\alpha}$ $\sim m_{\tilde{\alpha}}(1 \leqq \alpha \leqq \mu)$ by an admissible permutation $\alpha \mapsto \tilde{\alpha}$, is called an inessential change of the grading of $\mathscr{S}$. S-systems $\mathscr{S}=\left(S ;\left\{m_{\alpha}\right\}\right)$ of rank $\mu$ and $\mathscr{S}^{\prime}=\left(S^{\prime} ;\left\{m_{\alpha}^{\prime}\right\}\right)$ of rank $\mu^{\prime}$ are said to be equivalent, if (1) $\mu=\mu^{\prime}$ and (2) after an inessential change of the grading of $\mathscr{S}, m_{\alpha}$ $=m_{\alpha}^{\prime}(1 \leqq \alpha \leqq \mu)$ and there exists an algebra isomorphism of $S$ onto $S^{\prime}$, 
which preserves gradings and commutes with involutions, $j$ and $\mathscr{P}$. The equivalence class of $\mathscr{S}$ will be denoted by $\{\mathscr{S}\}$.

Let $C$ be an $S$-algebra of rank $m$. We define an equivalence relation $\sim$ in the set $I=\{1,2, \cdots, m\}$ inductively as follows: Let $i \sim i$ for each $i$. Suppose that an equivalence relation $i \sim k$ is defined for each $i, k$ with $|i-k|<p$. We define $i, k$ with $|i-k|=p$ to be $i \sim k$ if (1) $n_{i k} \neq 0$ and $n_{i, m+1}=n_{k, m+1}$; (2) $n_{i \ell}=n_{k \ell}$ for each $\ell \neq i$, $k$; (3) for each $s$ lying between $i$ and $k$ (except $i$ and $k$ ), $n_{i s}=n_{k s}=0$ or $i \sim s$, $s \sim k$. A grading of $C$ is said to be adapted to the relation $\sim$, if $i<k$ $<\ell, i \sim \ell$ imply $i \sim k \sim \ell$. In this case we get the decomposition $I=$ $\bigcup_{\alpha=1}^{\mu} I_{\alpha}$ of $I$ into the $\sim$-equivalence classes $I_{\alpha}(1 \leqq \alpha \leqq \mu)$ in such a way that

$$
I_{\alpha}=\left[m_{1}+\cdots+m_{\alpha-1}+1, m_{1}+\cdots+m_{\alpha-1}+m_{\alpha}\right] \quad \text { for } 1 \leqq \alpha \leqq \mu,
$$

and hence

$$
m=\sum_{\alpha=1}^{\mu} m_{\alpha}
$$

We put

$$
I_{\mu+1}=\{m+1\}, \quad m_{\mu+1}=1 .
$$

Then the cardinality $\left|I_{\alpha}\right|$ of $I_{\alpha}$ is equal to $m_{\alpha}$ for each $1 \leqq \alpha \leqq \mu+1$. We define $\alpha(i)$ with $1 \leqq \alpha(i) \leqq \mu+1$ by the relation

$$
i \in I_{\alpha(i)} \quad(1 \leqq i \leqq m+1) .
$$

THEOREM 2.1. (A) For each S-algebra $C$, we can make the grading of $C$ adapted to the relation $\sim$, by an inessential change of the grading of $C$.

(B) Let $C$ be an S-algebra of rank $m$ with the grading adapted to the relation $\sim$. Then we can associate to $C$ an $S$-system $\mathscr{S}=$ $\left(S ;\left\{m_{\alpha}\right\}_{1 \leqq \alpha \leqq \mu+1}\right)$ of rank $\mu$ with the following properties:

(a) $\mu$ is the number of the -equivalence classes $I_{\alpha}$ in $I=\{1,2$, $\cdots, m\}$, and $m_{\alpha}=\left|I_{\alpha}\right|(1 \leqq \alpha \leqq \mu)$.

(b) If $\alpha \neq \beta$ or $\beta \neq \gamma$,

$$
\begin{aligned}
& u_{\alpha \alpha}\left(v_{\alpha \beta} w_{\beta \gamma}\right)=\left(u_{\alpha \alpha} v_{\alpha \beta}\right) w_{\beta \gamma}, \\
& u_{\alpha \beta}\left(v_{\beta \beta} w_{\beta \gamma}\right)=\left(u_{\alpha \beta} v_{\beta \beta}\right) w_{\beta r} .
\end{aligned}
$$


(c) There exist linear isomorphisms

$$
\theta^{i k}: C_{i k} \longrightarrow \begin{cases}S_{\alpha(i) \alpha(k)} & \text { if } \alpha(i) \neq \alpha(k) \\ \mathscr{P} S_{\alpha(i) \alpha(i)} & \text { if } \alpha(i)=\alpha(k), i \neq k \\ \mathscr{R} e S_{\alpha(i) \alpha(i)} & \text { if } i=k\end{cases}
$$

satisfying

$$
\theta^{i \ell}\left(a_{i k} b_{k \ell}\right)= \begin{cases}\theta^{i k}\left(a_{i k}\right) \theta^{k \ell}\left(b_{k \ell}\right) & \text { if } \alpha(i) \neq \alpha(\ell) \\ \mathscr{P}\left(\theta^{i k}\left(a_{i k}\right) \theta^{k \ell}\left(b_{k \ell}\right)\right) & \text { if } \alpha(i)=\alpha(\ell), i \neq \ell \\ \mathscr{R} e\left(\theta^{i k}\left(a_{i k}\right) \theta^{k \ell}\left(b_{k \ell}\right)\right) & \text { if } i=\ell\end{cases}
$$

and hence $\theta^{i i}=\theta$ for each $i$,

$$
\begin{gathered}
\theta^{i k}\left(a_{k i}^{*}\right)=\left(\theta^{k i}\left(a_{k i}\right)\right)^{*}, \\
j \theta^{i, m+1}\left(a_{i, m+1}\right)=\theta^{i, m+1}\left(j a_{i, m+1}\right), \quad j \theta^{m+1, i}\left(a_{m+1, i}\right)=\theta^{m+1, i}\left(j a_{m+1, i}\right) \\
\text { for each } 1 \leqq i \leqq m .
\end{gathered}
$$

Hence we have

$$
\operatorname{dim} C_{i k}= \begin{cases}\operatorname{dim} S_{\alpha(i) \alpha(k)} & \text { if } \alpha(i) \neq \alpha(k) \\ \nu_{\alpha(i)} & \text { if } \alpha(i)=\alpha(k), i \neq k \\ 1 & \text { if } i=k .\end{cases}
$$

(C) We associate to each S-algebra $C$ an S-system $\mathscr{S}$ by the procedures $(A)$ and $(B)$. Then the correspondence $C \backsim \mathscr{S}$ induces an injective map from the set of isomorphism classes of S-algebras into the set of equivalence classes of S-systems.

Proof. We give the proof of the injectivity of the last map, since the proof of other assertions was contained in Takeuchi [7].

Let $\mathscr{S}=\left(S ;\left\{m_{\alpha}\right\}_{1 \leqq \alpha \leqq \mu+1}\right)$ be an $S$-system. We define $m, I_{\alpha}(1 \leqq \alpha$ $\leqq \mu+1)$ and $\alpha(i) \quad(1 \leqq i \leqq m+1)$ by $(2.1) \sim(2.4)$. Take real vector spaces $C_{i k}(1 \leqq i, k \leqq m+1)$ with dimensions (2.6) and linear isomorphisms $\theta^{i k}$ as in (2.5). Put

$$
C=\sum_{1 \leqq i, k \leqq m+1} C_{i k}
$$

We define a product $a b$, an involutive linear automorphism $a \mapsto a^{*}$ and a partial complex structure $j$ on $C$ by the relations (1), (2) and (3). It is easy to see that $a \mapsto a^{*}$ is an anti-automorphism of $C$, making use of the commutativity of $u \mapsto u^{*}$ with $\mathscr{P}$ and $\mathscr{R} e$, and hence $C$ becomes a 
matrix algebra with involution and partial complex structure. If furthermore $\mathscr{S}$ is associated to an $S$-algebra $C^{\prime}$, then $C$ becomes an $S$ algebra, which is isomorphic to $C^{\prime}$. This shows the injectivity of our map.

q.e.d.

For an $S$-algebra $C$ of the grading adapted to the relation $\sim$, the subalgebra

$$
C^{c}=\sum_{\alpha(i)=\alpha(k)} C_{i k}
$$

of $C$ is called the kernel of $C$. We have the following multiplicative properties related to the kernel $C^{c}$ (Takeuchi [7]).

Lemma. 1) If $i \neq p, k \neq q$ and one of $a_{i k}, b_{k p}, c_{p q}$ belongs to $C^{c}$, then

$$
a_{i k}\left(b_{k p} c_{p q}\right)=\left(a_{i k} b_{k p}\right) c_{p q} .
$$

2) If $i \neq \ell$ and $\alpha(k)$ equals $\alpha(i)$ or $\alpha(\ell)$, then

$$
\begin{aligned}
& a_{i k}\left(b_{k \ell} b_{k \ell}^{*}\right)=\left(a_{i k} b_{k l}\right) b_{k \ell}^{*}, \\
& a_{i k}^{*}\left(a_{i k} b_{k \ell}\right)=\left(a_{i k}^{*} a_{i k}\right) b_{k \ell},
\end{aligned}
$$

or equivalently,

$$
\begin{aligned}
& a_{i k}\left(b_{k \ell} c_{k \ell}^{*}\right)+a_{i k}\left(c_{k \ell} b_{k \ell}^{*}\right)=\left(a_{i k} b_{k \ell}\right) c_{k \ell}^{*}+\left(a_{i k} c_{k \ell}\right) b_{k \ell}^{*}, \\
& a_{i k}^{*}\left(b_{i k} c_{k \ell}\right)+b_{i k}^{*}\left(a_{i k} c_{k \ell}\right)=\left(a_{i k}^{*} b_{i k}\right) c_{k \ell}+\left(b_{i k}^{*} a_{i k}\right) c_{k \ell} .
\end{aligned}
$$

3) If $1 \leqq i \leqq m$ and $a_{i k} \in C^{c}$, then

$$
\begin{aligned}
a_{i k}\left(j b_{k, m+1}\right) & =j\left(a_{i k} b_{k, m+1}\right), \\
\left(j b_{m+1, i}\right) a_{i k} & =j\left(b_{m+1, i} a_{i k}\right) .
\end{aligned}
$$

Now we consider the group of linear automorphisms of Siegel domains. Let $D(V, F) \subset X^{c} \times Y$ be a Siegel domain. Put

$$
G L(D(V, F))=A(D(V, F)) \cap G L\left(X^{c} \times Y\right) .
$$

Let $g r(D(V, F))$ and $g(V)$ denote the Lie algebras of $G L(D(V, F))$ and $G(V)$ respectively. Identifying as $\mathfrak{g r}(X) \oplus \mathfrak{g r}(Y) \subset \mathfrak{g r}\left(X^{c} \times Y\right)$, we have (PjateckiiŠapiro [5])

$$
\begin{aligned}
\operatorname{gr}(D(V, F)) & =\left\{\left(\psi^{\prime}, \psi^{\prime \prime}\right) \in \mathfrak{g}(V) \oplus \operatorname{gr}(Y) ; \psi^{\prime} F(u, v)\right. \\
& \left.=F\left(\psi^{\prime \prime} u, v\right)+F\left(u, \psi^{\prime \prime} v\right)(u, v \in Y)\right\}
\end{aligned}
$$


The projection $\pi_{X}: \mathfrak{g l}(D(V, F)) \rightarrow \mathfrak{g}(V)$ is defined by the correspondence $\left(\psi^{\prime}, \psi^{\prime \prime}\right) \mapsto \psi^{\prime}$. In particular, for the Siegel domain $D(C) \subset X^{c} \times(Y, j)$ associated to an $S$-algebra $C$, we have an identification

$$
\begin{aligned}
& \operatorname{gl}(D(C))=\left\{\left(\psi^{\prime}, \psi^{\prime \prime}\right) \in \mathfrak{g}(V(A)) \oplus \mathfrak{g} \mathfrak{l}(Y) ; \psi^{\prime \prime} j=j \psi^{\prime \prime},\right. \\
& \left.\psi^{\prime} \Phi(u, v)=\Phi\left(\psi^{\prime \prime} u, v\right)+\Phi\left(u, \psi^{\prime \prime} v\right) \quad(u, v \in Y)\right\},
\end{aligned}
$$

where $\Phi: Y \times Y \rightarrow X$ is the symmetric map associated to $C$. It is known (Vinberg-Gindikin-Pjateckii-Šapiro [12]) that $\mathfrak{g l}(D(C)$ ) (resp. $g(V(A))$ ) is an algebraic Lie algebra in $\mathfrak{g l}(X \times Y)$ (resp. in $\mathfrak{g l}(X)$ ), and $\pi_{X}: \mathfrak{g l}(D(C)$ ) $\rightarrow \mathrm{g}(V(A))$ is a rational homomorphism. We shall describe the structure of $\operatorname{gr}(D(C))$ in terms of the $S$-algebra $C$. Assume that $C$ has the grading adapted to the relation $\sim$. Put

$$
X^{c}=C^{c} \cap X, \quad V^{c}=C^{c} \cap V(A) .
$$

Then it can be shown that $V^{c}$ is the direct product $V_{1} \times \cdots \times V_{\mu}$ of the cones $V_{\alpha}$ corresponding to the $T$-algebras

$$
A_{\alpha}=\sum_{\alpha(i)=\alpha(k)=\alpha} C_{i k} \quad(1 \leqq \alpha \leqq \mu),
$$

and hence $V^{c}$ is a self-dual homogeneous convex cone in $X^{c}$, in virtue of the characterization of Vinberg cited in $\S 1$. The Lie algebra $g\left(V^{c}\right)$ of $G\left(V^{c}\right)$ has a Cartan decomposition

$$
\mathfrak{g}\left(V^{c}\right)=\mathfrak{H}\left(V^{c}\right)+\mathfrak{p}\left(V^{c}\right),
$$

where $\mathfrak{f}\left(V^{c}\right)$ is the stabilizer of $e_{A}=\sum_{1 \leqq i \leqq m} e_{i} \in V^{c}$ in $\mathfrak{g}\left(V^{c}\right)$, and $\mathfrak{p}\left(V^{c}\right)$ is the image $\psi^{c}\left(X^{c}\right)$ of the injective linear map $\psi^{c}: X^{c} \rightarrow \operatorname{gr}\left(X^{c}\right)$ defined by

$$
\psi^{c}(x) y=x y+y x \quad \text { for } x, y \in X^{c} .
$$

We define linear maps $\psi^{\prime}: X^{c} \rightarrow \mathfrak{g l}(X)$ and $\psi^{\prime \prime}: X^{c} \rightarrow \mathfrak{g} \mathfrak{l}(Y)$ by

$$
\begin{aligned}
\psi^{\prime}(x) y=x y+y x & \text { for } x \in X^{c}, y \in X, \\
\psi^{\prime \prime}(x) u=x u+u x & \text { for } x \in X^{c}, u \in Y,
\end{aligned}
$$

and then define an injective linear map $\psi: X^{c} \rightarrow \operatorname{gl}(X) \oplus \mathfrak{g l}(Y)$ by $\psi=$ $\psi^{\prime} \oplus \psi^{\prime \prime}$, i.e.,

$$
\psi(x)(y, u)=\left(\psi^{\prime}(x) y, \psi^{\prime \prime}(x) u\right) \quad \text { for } x \in X^{c}, y \in X, u \in Y .
$$

Put 


$$
\boldsymbol{T}^{u}=\sum_{1 \leqq \alpha(i)<\alpha(k) \leqq \mu} C_{i k} .
$$

Then $T^{u}$ becomes a Lie algebra by the bracket product $\left[t, t^{\prime}\right]=t t^{\prime}-t^{\prime} t$. A faithful representation $\psi: T^{u} \rightarrow \operatorname{gr}(X) \oplus \operatorname{gr}(Y)$ is defined by

$$
\psi(t)(x, u)=\left(t x+x t^{*}, t u+u t^{*}\right) \quad \text { for } t \in T^{u}, x \in X, u \in Y .
$$

We define subspaces $\mathfrak{p}^{c}$ and $\mathfrak{t}^{u}$ of $\mathfrak{g l}(X) \oplus \mathfrak{g l}(Y)$ by

$$
\mathfrak{p}^{c}=\psi\left(X^{c}\right), \quad t^{u}=\psi\left(\boldsymbol{T}^{u}\right) .
$$

$t^{u}$ is nilpotent in the sense that it consists of nilpotent endomorphisms of $X \times Y$. Then we have

THEOREM 2.2. (Takeuchi [7]) We have a direct sum decomposition

$$
\mathfrak{g} \mathfrak{l}(D(C))=\mathfrak{g}_{0}+\mathfrak{g}^{c}+\mathfrak{t}^{u}
$$

as vector spaces with the following properties:

1) $t^{u}$ is the maximum nilpotent ideal of $\operatorname{gr}(D(C))$.

2) $\mathrm{g}^{c}$ is a reductive algebraic subalgebra of $\mathrm{g}((D(C))$ without compact factors. $\mathrm{g}^{c} X^{c} \subset X^{c}$ and the restriction to $X^{c}$ induces an isomorphism of $\mathrm{g}^{c}$ onto $\mathrm{g}\left(V^{c}\right)$. We have a Cartan decomposition

$$
\mathfrak{g}^{c}=\mathfrak{f}^{c}+\mathfrak{p}^{c},
$$

where $\mathfrak{i}^{c}$ is the stabilizer of $e_{A}$ in $\mathrm{g}^{c}$, and $\mathfrak{p}^{c}$ is the one defined in the above.

3) $\mathfrak{g}_{0}$ is a compact subalgebra of $\mathfrak{g}\left(D(C)\right.$ ) satisfying $\mathfrak{g}_{0} X^{c}=\{0\}$ and $\left[g_{0}, g^{c}\right]=\{0\}$.

4) The stabilizer $\mathfrak{f}$ of $e_{A}$ in $\operatorname{gr}(D(C))$ is a maximal compact subalgebra of $\operatorname{gr}(D(C))$ with the decomposition

$$
\mathfrak{H}=\mathfrak{g}_{0} \oplus \mathfrak{l}^{\mathfrak{c}} .
$$

CoRollary. Let $C$ be an S-algebra. Then the rank of an S-system associated to $C$ is equal to 1 if and only if (1) the associated T-algebra $A$ is isomorphic to one of the $A_{m}^{\nu}$, and (2) the projection $\pi_{X}: \operatorname{gr}(D(C)) \rightarrow$ $\mathrm{g}(V(A))$ is surjective.

Proof. We may assume that $C$ has the grading adapted to the relation $\sim$. Let $\mathscr{S}=\left(S ;\left\{m_{\alpha}\right\}_{1 \leqq \alpha \leqq u+1}\right)$ be an $S$-system associated to $C$.

Assume $\mu=1$. Then $T^{u}=\{0\}, X=X^{c}, V(A)=V^{c}$ and $A$ is isomorphic to $A_{m_{1}}^{\nu_{1}}$. It follows from Theorem 2.2 that 


$$
\mathfrak{g l}(D(C))=\mathfrak{g}_{0} \oplus \mathfrak{g}^{c},
$$

where $\mathfrak{g}_{0} X=\{0\}$ and $\mathfrak{g}^{c}$ is isomorphic to $\mathfrak{g}(V(A))$ through the restriction to $X$, and hence $\pi_{X}$ is surjective.

Conversely assume that (1) and (2) are satisfied. It follows from (2) that $\pi_{X}\left(\mathrm{~g}((D(C)))\right.$ is reductive and $\pi_{X}\left(\mathrm{t}^{u}\right)$ is a nilpotent ideal in $\pi_{X}(\mathfrak{g}(D(C)))$, and hence $\pi_{X}\left(t^{u}\right)=\{0\}$. Since $\pi_{X}$ is injective on $t^{u}$, we have $T^{u}=\{0\}$. Now (1) implies $\mu=1$.

q.e.d.

\section{Siegel domains satisfying (i) and (ii)}

Lemma 1. Let $D(V, F) \subset X^{c} \times Y$ be a Siegel domain associated to a self-dual homogeneous convex cone $V$. Then the Satake's second condition (ii) is equivalent to the following condition

(ii) $^{\prime} \pi_{X}: \operatorname{gr}(D(V, F)) \rightarrow \mathrm{g}(V)$ is surjective.

If $D(V, F)$ satisfies (ii), it is affinely homogeneous.

Proof. If the condition (ii) is satisfied, we have $\mathfrak{p}(V) \subset \pi_{X}(\mathfrak{g} \mathfrak{l}(D(V, F)))$ in the notation in Introduction. On the other hand, we have $g(V)=$ $\mathfrak{f}(V)+\mathfrak{p}(V)$ with $[\mathfrak{p}(V), \mathfrak{p}(V)]=\mathfrak{f}(V)$, and hence $\mathfrak{g}(V) \subset \pi_{X}(\mathfrak{g} l(D(V, F)))$. This shows (ii)'.

Conversely assume (ii)'. Then $D(V, F)$ is affinely homogeneous (cf. Pjateckii-Šapiro [5]). From the arguments in $\S 1$, we may assume that $D(V, F)=D(C)$ of an $S$-algebra $C$ of rank $m$ satisfying (1) the associated $T$-algebra $A=A_{m}^{\nu}$, and (2) $\pi_{X}: \mathfrak{g l}(D(C)) \rightarrow \mathfrak{g}\left(V_{m}^{\nu}\right)$ is surjective. Then as we have seen in Theorem 2.2 and Cor., $\mathfrak{g} \mathfrak{l}(D(C))$ contains the image $\mathfrak{p}^{c}$ $=\psi\left(X_{m}^{\nu}\right)$ of the map $\psi=\psi^{\prime} \oplus \psi^{\prime \prime}$ given by

$$
\begin{aligned}
\psi^{\prime}(x) y=x y+y x & \text { for } x, y \in X_{m}^{v}=X, \\
\psi^{\prime \prime}(x) u=x u+u x & \text { for } x \in X_{m}^{\nu}, u \in Y .
\end{aligned}
$$

Take the base point $e_{A} \in V_{m}^{\nu}$ and the standard inner product (, ) on $X_{m}^{\nu}$. Recall that then

$$
T(x)=\frac{1}{2} \psi^{\prime}(x) \quad \text { for } x \in X_{m}^{\nu} .
$$

We shall show that if we put

$$
R(x)=\frac{1}{2} \psi^{\prime \prime}(x) \quad \text { for } x \in X_{m}^{\nu},
$$

$R(x)$ is self-adjoint with respect to the hermitian inner product $\left(e_{A}, F(u, v)\right)$ on $(Y, j)$; this will imply (ii). We may assume $n_{i}=1$ for $1 \leqq i \leqq m$, 
in virtue of Remark of $\S 1$. Thus the inner product $($,$) on C$ coincides on $X_{m}^{\nu}$ with the standard inner product of $X_{m}^{\nu}$. We define a hermitian inner product $\langle$,$\rangle on (Y, j)$ by

$$
\langle u, v\rangle=(u, v)+\sqrt{-1}(u, j v) \quad \text { for } u, v \in Y .
$$

Then we have $4\left(e_{A}, F(u, v)\right)=\langle u, v\rangle$ for each $u, v \in Y$. In fact, for the symmetric map $\Phi$ associated to $C$ we have

$$
\begin{aligned}
2\left(e_{A}, \Phi(u, v)\right) & =\left(e_{A}, \hat{u} v+\hat{v} u\right)=\left(v^{*}, \hat{u}\right)+\left(\hat{v}^{*}, u\right) \\
& =(\hat{u}, \hat{v})+(\underline{u}, v)=(u, v) .
\end{aligned}
$$

Furthermore we have

$$
\begin{aligned}
\left(\psi^{\prime \prime}(x) u, v\right) & =(x u+u x, v)=\left(u, x^{*} v\right)+\left(u, v x^{*}\right) \\
& =(u, x v+v x)=\left(u, \psi^{\prime \prime}(x) v\right) \quad \text { for } x \in X_{m}^{\nu}, u, v \in Y,
\end{aligned}
$$

and hence $\psi^{\prime \prime}(x)$ is self-adjoint with respect to the hermitian inner product $\langle$,$\rangle . This implies the required assertion.$

The last assertion of the Lemma is clear from the above arguments.

q.e.d.

Lemma 2. Let $\mathscr{S}=\left(S ;\left\{m_{\alpha}\right\}_{1 \leqq \alpha \leqq 2}\right)$ be an $S$-system of rank $\mu=1$. Then $\mathscr{S}$ is associated to an S-algebra if and only if it satisfies the following conditions 1) 8):

1) $\varepsilon_{\alpha} u_{\alpha \beta}=u_{\alpha \beta}$.

2) There exist $\ell_{\alpha}>0(1 \leqq \alpha \leqq \mu+1)$ such that

$$
\ell_{\alpha} \mathscr{R}_{e}\left(u_{\alpha \beta} v_{\beta \alpha}\right)=\ell_{\beta} \mathscr{R}_{e}\left(v_{\beta \alpha} u_{\alpha \beta}\right) \text {. }
$$

3) $\mathscr{R}_{e}\left(u_{\alpha \beta} u_{\alpha \beta}^{*}\right)>0$ if $u_{\alpha \beta} \neq 0$.

4) $\mathscr{R}_{e}\left(u_{\alpha \beta}\left(v_{\beta r} w_{r \alpha}\right)\right)=\mathscr{R} e\left(\left(u_{\alpha \beta} v_{\beta r}\right) w_{r \alpha}\right)$.

5) $u_{\alpha \beta}\left(j v_{\beta, \mu+1}\right)=j\left(u_{\alpha \beta} v_{\beta, \mu+1}\right)$ if $1 \leqq \alpha \leqq \beta \leqq \mu$.

6) $j\left(u_{\alpha, \mu+1}^{*}\right)=\left(j u_{\alpha, \mu+1}\right) *$ if $1 \leqq \alpha \leqq \mu$.

7) $\mathscr{R} e\left(j u_{\alpha, \mu+1}\left(j u_{\alpha, \mu+1}\right) *\right)=\mathscr{R} e\left(u_{\alpha, \mu+1} u_{\alpha, \mu+1}^{*}\right)$ if $1 \leqq \alpha \leqq \mu$.

8) 8-1) $\lambda(\mu u)=(\lambda \mu) u$ for $\lambda, \mu \in S_{11}, u \in S_{12}$,

8-2) $\lambda(u v)=(\lambda u) v$ for $\lambda \in S_{11}, u \in S_{12}, v \in S_{21}$.

Remark 1. For a general $S$-system $\mathscr{S}=\left(S ;\left\{m_{\alpha}\right\}_{1 \leqq \alpha \leqq \mu+1}\right)$ of rank $\mu$, the conditions in order that $\mathscr{S}$ is associated to an $S$-algebra, are given by the above conditions 1) 7) for general $\mu$, together with the following associativity conditions: 
8) If $\alpha \neq \beta$ or $\beta \neq \gamma$,

$$
\begin{aligned}
& u_{\alpha \alpha}\left(v_{\alpha \beta} w_{\beta \gamma}\right)=\left(u_{\alpha \alpha} v_{\alpha \beta}\right) w_{\beta \gamma}, \\
& u_{\alpha \beta}\left(v_{\beta \beta} w_{\beta \gamma}\right)=\left(u_{\alpha \beta} v_{\beta \beta}\right) w_{\beta \gamma} .
\end{aligned}
$$

9) $u_{\alpha \beta}\left(v_{\beta \gamma} w_{\gamma \delta}\right)=\left(u_{\alpha \beta} v_{\beta \gamma}\right) w_{r \delta}$ if $\alpha<\beta<\gamma$ and $\beta<\delta$.

10) $u_{\alpha \beta} \mathscr{P}\left(v_{\beta r} w_{\gamma \beta}\right)=\left(u_{\alpha \beta} v_{\beta r}\right) w_{\gamma \beta}$ if $\alpha<\beta<\gamma$ and $m_{\beta} \geqq 2$, $u_{\alpha \beta}\left(v_{\beta \gamma} v_{\beta \gamma}^{*}\right)=\left(u_{\alpha \beta} v_{\beta \gamma}\right) v_{\beta \gamma}^{*}$ if $\alpha<\beta<\gamma$ and $m_{\beta}=1$.

Proof of Lemma 2. Let $\mathscr{S}$ be associated as in Theorem 2.1, (B) to an $S$-algebra of the grading adapted to the relation $\sim$. Then 1 ) follows from the axiom (II) of $S$-algebras. In virtue of Remark of $\S 1$, we may assume that $n_{i}=n_{k}$ if $\alpha(i)=\alpha(k)$. Put $\ell_{\alpha(i)}=n_{i}$. Then 2) follows from the axiom (III) and the equality $(\lambda, \mu)=(\bar{\lambda}, \bar{\mu})$ for the standard inner product $\left(\right.$, ) of $\boldsymbol{F}_{m}^{\nu}$, seeing that $\boldsymbol{F}_{m}^{\nu}$ is generated by $\mathscr{P} \boldsymbol{F}_{m}^{\nu}$. We get 3) making use of the axiom (IV). Theorem 2.1, (B), (b) implies 8-1), 8-2) and

$$
\text { 8-3) } u(\lambda v)=(u \lambda) v \quad \text { for } u \in S_{21}, v \in S_{12}, \lambda \in S_{11} .
$$

Applying the involution $u \mapsto u^{*}$ on $8-2$ ), we get

$$
\text { 8-2) } \quad(u v) \lambda=u(v \lambda) \quad \text { for } u \in S_{12}, v \in S_{21}, \lambda \in S_{11} .
$$

Now 4) follows from 8-3), 8-2)' and the equality $(\lambda \mu, \nu)=(\mu, \bar{\lambda} \nu)$ for the standard inner product of $F_{m}^{\nu}$. 5) follows from the axiom (VIII), seeing also that $\boldsymbol{F}_{m}^{\nu}$ is generated by $\mathscr{P} \boldsymbol{F}_{m}^{\nu}$. 6) and 7) follow from the axioms (IX) and (X) respectively.

Conversely assume that $\mathscr{S}$ satisfies the conditions 1) $\sim$ ). Let

$$
C=\sum_{1 \leqq i, k \leqq m+1} C_{i k}, \quad m=m_{1}
$$

be the matrix algebra with involution $a \mapsto a^{*}$ and partial complex structure $j$, constructed from $\mathscr{S}$ as in $(C)$ of Theorem 2.1. We shall prove axioms $(\mathrm{I}) \sim(X)$ for $C$.

(I) Put $\theta=\theta^{i i}: C_{i i} \rightarrow \boldsymbol{R} \varepsilon_{\alpha(i)}=\boldsymbol{R}$. Then

$$
\theta^{i i}\left(a_{i i} b_{i i}\right)=\mathscr{R} e\left(\theta^{i i}\left(a_{i i}\right) \theta^{i i}\left(b_{i i}\right)\right)=\theta^{i i}\left(a_{i i}\right) \theta^{i i}\left(b_{i i}\right)
$$

implies that $\theta$ is an algebra isomorphism.

(II) The unit element $e_{i} \in C_{i i}$ satisfies $\theta^{i i}\left(e_{i}\right)=\varepsilon_{\alpha(i)}$. It follows from 1) 


$$
\begin{aligned}
\theta^{i k}\left(e_{i} a_{i k}\right) & = \begin{cases}\theta^{i i}\left(e_{i}\right) \theta^{i k}\left(a_{i k}\right)=\theta^{i k}\left(a_{i k}\right) & \text { if } \alpha(i) \neq \alpha(k) \\
\mathscr{P}\left(\theta^{i i}\left(e_{i}\right) \theta^{i k}\left(a_{i k}\right)\right)=\mathscr{P} \theta^{i k}\left(a_{i k}\right) & \text { if } \alpha(i)=\alpha(k), i \neq k \\
\mathscr{R e}\left(\theta^{i i}\left(e_{i}\right) \theta^{i k}\left(a_{i k}\right)\right)=\mathscr{R} e \theta^{i k}\left(a_{i k}\right) & \text { if } i=k\end{cases} \\
& =\theta^{i k}\left(a_{i k}\right) .
\end{aligned}
$$

This implies $e_{i} a_{i k}=a_{i k}$.

(III) Put $n_{i}=\ell_{\alpha(i)}$. Then from 2) it follows

$$
\begin{aligned}
n_{i} \theta\left(a_{i k} b_{k i}\right) & =\ell_{\alpha(i)} \theta^{i i}\left(a_{i k} b_{k i}\right)=\ell_{\alpha(i)} \mathscr{R}_{e}\left(\theta^{i k}\left(a_{i k}\right) \theta^{k i}\left(b_{k i}\right)\right) \\
& =\ell_{\alpha(k)} \mathscr{R}_{e}\left(\theta^{k i}\left(b_{k i}\right) \theta^{i k}\left(a_{i k}\right)\right)=\ell_{\alpha(k)} \theta^{k k}\left(b_{k i} a_{i k}\right) \\
& =n_{k} \theta\left(b_{k i} a_{i k}\right) .
\end{aligned}
$$

(IV) If $a_{i k} \neq 0$,

$$
\begin{aligned}
\theta\left(a_{i k} a_{i k}^{*}\right) & =\theta^{i i}\left(a_{i k} a_{i k}^{*}\right)=\mathscr{R} e\left(\theta^{i k}\left(a_{i k}\right) \theta^{k i}\left(a_{i k}^{*}\right)\right) \\
& =\mathscr{R e}\left(\theta^{i k}\left(a_{i k}\right) \theta^{i k}\left(a_{i k}\right)^{*}\right)>0
\end{aligned}
$$

in virtue of 3 ).

(V): $\quad a_{i k}\left(b_{k \ell} c_{\ell i}\right)=\left(a_{i k} b_{k \ell}\right) c_{\ell i}$. We may assume that $i, k$, $\ell$ are mutually distinct, in virtue of (II). Then

$$
\begin{aligned}
& \theta^{i i}\left(a_{i k}\left(b_{k \ell} c_{\ell i}\right)\right)=\mathscr{R}_{e}\left(\theta^{i k}\left(a_{i k}\right) \theta^{k i}\left(b_{k \ell} c_{\ell i}\right)\right) \\
& = \begin{cases}\mathscr{R}_{e}\left[\theta^{i k}\left(a_{i k}\right)\left(\theta^{k \ell}\left(b_{k \ell}\right) \theta^{\ell i}\left(c_{\ell i}\right)\right)\right] & \text { if } \alpha(k) \neq \alpha(i) \\
\mathscr{R}_{e}\left[\theta^{i k}\left(a_{i k}\right) \mathscr{P}\left(\theta^{k \ell}\left(b_{k \ell}\right) \theta^{\ell i}\left(c_{\ell i}\right)\right)\right] & \text { if } \alpha(k)=\alpha(i)\end{cases} \\
& =\mathscr{R} e\left[\theta^{i k}\left(a_{i k}\right)\left(\theta^{k \ell}\left(b_{k \ell}\right) \theta^{\ell i}\left(c_{\ell i}\right)\right)\right] \text {, }
\end{aligned}
$$

since $\mathscr{R}_{e}(\lambda \mu)=\mathscr{R}_{e}(\lambda \mathscr{P} u)$ for $\lambda \in \mathscr{P} \boldsymbol{F}_{m}^{\nu}, \mu \in \boldsymbol{F}_{m}^{\nu}$. In the same way we get

$$
\theta^{i i}\left(\left(a_{i k} b_{k \ell}\right) c_{\ell i}\right)=\mathscr{R}_{e}\left[\left(\theta^{i k}\left(a_{i k}\right) \theta^{k \ell}\left(b_{k \ell}\right)\right) \theta^{\ell i}\left(c_{\ell i}\right)\right] .
$$

Now (V) follows from 4).

(VI): $\quad a_{i k}\left(b_{k \ell} c_{\ell p}\right)=\left(a_{i k} b_{k \ell}\right) c_{\ell p}$ if $i<k<\ell, k<p . \quad$ In virtue of (II), we may consider the following three cases:

(a) $\alpha(i)=\alpha(k)=\alpha(\ell)=1<\alpha(p)=2, m \geqq 3$,

(b) $\quad \alpha(i)=\alpha(k)=\alpha(p)=1<\alpha(\ell)=2, m \geqq 3$,

(c) $\quad \alpha(i)=\alpha(k)=\alpha(\ell)=\alpha(p)=1, \ell \neq p, m \geqq 4$.

In case (a), we have

$$
\begin{aligned}
\theta^{i p}\left(a_{i k}\left(b_{k \ell} c_{\ell p}\right)\right) & =\theta^{i k}\left(a_{i k}\right) \theta^{k p}\left(b_{k \ell} c_{\ell p}\right)=\theta^{i k}\left(a_{i k}\right)\left(\theta^{k \ell}\left(b_{k \ell}\right) \theta^{\ell p}\left(c_{\ell p}\right)\right) \\
& \left.=\left(\theta^{i k}\left(a_{i k}\right) \theta^{k \ell}\left(b_{k \ell}\right)\right) \theta^{\ell p}\left(c_{\ell p}\right) \quad \text { by } 8-1\right) \\
& =\theta^{i \ell}\left(a_{i k} b_{k \ell}\right) \theta^{\ell p}\left(c_{\ell p}\right) \quad \text { since } m \neq 2 \\
& =\theta^{i p}\left(\left(a_{i k} b_{k \ell}\right) c_{\ell p}\right) .
\end{aligned}
$$


This implies (VI). In case (b), making use of $8-2$ ) and $m \neq 2$, we have

$$
\theta^{i p}\left(a_{i k}\left(b_{k \ell} c_{\ell p}\right)\right)=\theta^{i p}\left(\left(a_{i k} b_{k \ell}\right) c_{\ell p}\right),
$$

which implies (VI). In case (c), making use of $m \neq 2$, we have

$$
\begin{aligned}
\theta^{i p}\left(a_{i k}\left(b_{k \ell} c_{\ell p}\right)\right) & =\theta^{i k}\left(a_{i k}\right)\left(\theta^{k \ell}\left(b_{k \ell}\right) \theta^{\ell p}\left(c_{\ell p}\right)\right), \\
\left.\theta^{i p}\left(a_{i k} b_{k \ell}\right) c_{\ell p}\right) & =\left(\theta^{i k}\left(a_{i k}\right) \theta^{k \ell}\left(b_{k \ell}\right)\right) \theta^{\ell p}\left(c_{\ell p}\right) .
\end{aligned}
$$

Since $\boldsymbol{F}_{m}^{\nu}$ is an associative algebra for $m \geqq 4$, we get (VI).

(VII) : $a_{i k}\left(b_{k \ell} b_{k \ell}^{*}\right)=\left(a_{i k} b_{k \ell}\right) b_{k \ell}^{*}$ if $i<k<\ell$. We may consider following two cases:

(a) $\alpha(i)=\alpha(k)=1<\alpha(\ell)=2, m \geqq 2$,

(b) $\alpha(i)=\alpha(k)=\alpha(\ell)=1, m \geqq 3$.

In case (a), we have

$$
\begin{aligned}
\left.\theta^{i k}\left(a_{i k}\left(b_{k \ell} b_{k \ell}^{*}\right)\right)\right) & =\mathscr{P}\left(\theta^{i k}\left(a_{i k}\right) \theta^{k k}\left(b_{k \ell} b_{k \ell}^{*}\right)\right) \\
& =\mathscr{P}\left[\theta^{i k}\left(a_{i k}\right) \mathscr{R} e\left(\theta^{k \ell}\left(b_{k \ell}\right) \theta^{k \ell}\left(b_{k \ell}\right) *\right)\right] \\
& =\mathscr{P}\left[\theta^{i k}\left(a_{i k}\right)\left(\theta^{k \ell}\left(b_{k \ell}\right) \theta^{k \ell}\left(b_{k \ell}\right)^{*}\right)\right],
\end{aligned}
$$

since $\mathscr{P}(\lambda \mathscr{R} e \mu)=\mathscr{P}(\lambda \mu)$ for $\lambda \in \mathscr{P} \boldsymbol{F}_{m}^{\nu}$ and $\mu \in \boldsymbol{F}_{m}^{\nu}$ with $\mu=\mu$. On the other hand

$$
\begin{aligned}
\theta^{i k}\left(\left(a_{i k} b_{k \ell}\right) b_{k \ell}^{*}\right) & =\mathscr{P}\left(\theta^{i \ell}\left(a_{i k} b_{k \ell}\right) \theta^{\ell k}\left(b_{k \ell}^{*}\right)\right) \\
& =\mathscr{P}\left[\left(\theta^{i k}\left(a_{i k}\right) \theta^{k \ell}\left(b_{k \ell}\right)\right) \theta^{k \ell}\left(b_{k \ell}\right)^{*}\right] \\
& =\mathscr{P}\left[\left(\theta^{i k}\left(a_{i k}\right)\left(\theta^{k \ell}\left(b_{k \ell}\right) \theta^{k \ell}\left(b_{k \ell}\right)^{*}\right)\right] \quad \text { by } 8-2\right) .
\end{aligned}
$$

These imply (VII). In case (b), we have

$$
\begin{aligned}
& \theta^{i k}\left(a_{i k}\left(b_{k \ell} b_{k \ell}^{*}\right)\right)=\theta^{i k}\left(a_{i k}\right)\left(\theta^{k \ell}\left(b_{k \ell}\right) \theta^{k \ell}\left(b_{k \ell}\right)^{*}\right), \\
& \theta^{i k}\left(\left(a_{i k} b_{k \ell}\right) b_{k \ell}^{*}\right)=\left(\theta^{i k}\left(a_{i k}\right) \theta^{k \ell}\left(b_{k \ell}\right)\right) \theta^{k \ell}\left(b_{k \ell}\right)^{*} .
\end{aligned}
$$

Together with the equality $\lambda(\mu \bar{\mu})=(\lambda \mu) \bar{\mu}$ in $\boldsymbol{F}_{m}^{\nu}$, we get (VII).

(VIII) follows from 5) and 8-1).

(IX) and (X) are immediate consequences of 6) and 7) respectively.

Remark 2. For an $S$-system $\left(S ;\left\{m_{\alpha}\right\}\right)$ of rank $\mu$ satisfying 2) and 3), we put

$$
\begin{aligned}
\operatorname{tr} u & =\sum_{\alpha} \ell_{\alpha} \mathscr{R} e u_{\alpha \alpha} & & \text { for } u=\left(u_{\alpha \beta}\right) \in S, \\
(u, v) & =\operatorname{tr} u v^{*} & & \text { for } u, v \in S .
\end{aligned}
$$


Then (, ) is an inner product on $S$ satisfying

$$
\left(u^{*}, v^{*}\right)=(u, v) \quad \text { for } u, v \in S,
$$

and the condition 4) is equivalent to

$$
(u v, w)=\left(v, u^{*} w\right) \quad \text { for } u, v, w \in S,
$$

or

$$
(u v, w)=\left(u, w v^{*}\right) \quad \text { for } u, v, w \in S .
$$

Let $\mathfrak{M}\left(\boldsymbol{F}_{m}^{\nu}\right)$ be the set of all equivalence classes of (finite dimensional and unital) $\boldsymbol{F}_{m}^{\nu}$-modules over $\boldsymbol{C}$, and $\mathfrak{M}^{*}\left(\boldsymbol{F}_{m}^{\nu}\right)$, Aut* $\left(\boldsymbol{F}_{m}^{\nu}, \mathscr{P}\right)$ and $\mathfrak{M}(m, \nu)$ be as in Introduction. Then the natural map from $\mathfrak{M} *\left(\boldsymbol{F}_{m}^{\nu}\right)$ into $\mathfrak{M}\left(\boldsymbol{F}_{m}^{\nu}\right)$ is a bijection. This can be proved in the same way as Lemma 21 in Ozeki-Takeuchi [4]. Since the natural map is equivariant with respect to the action of $\operatorname{Aut}^{*}\left(\boldsymbol{F}_{m}^{\nu}, \mathscr{P}\right)$, we have the identification

$$
\mathfrak{M}(m, \nu)=\operatorname{Aut}^{*}\left(\boldsymbol{F}_{m}^{\nu}, \mathscr{P}\right) \backslash \mathfrak{M} *\left(\boldsymbol{F}_{m}^{\nu}\right)=\operatorname{Aut}^{*}\left(\boldsymbol{F}_{m}^{\nu}, \mathscr{P}\right) \backslash \mathfrak{M}\left(\boldsymbol{F}_{m}^{\nu}\right)
$$

of orbit spaces. The point of $\mathfrak{M}(m, \nu)$ represented by an $\boldsymbol{F}_{m}^{\nu}$-module $W$ will be denoted by $[W]$.

In virtue of Lemma 1 and Cor. of Theorem 2.2, the classification of affine isomorphism classes of those Siegel domains $D(V, F)$ such that $V$ is an indecomposable self-dual homogeneous convex cone and it satisfies the condition (ii), is equivalent to the classification of isomorphism classes of those $S$-algebras such that the rank of an associated $S$-system is 1. Thus, in virtue of Theorem 2.1 and Lemma 2, our Theorem $\mathrm{A}$ in Introduction is equivalent to the following

THEOREM 3.1. The set $\subseteq(m, \nu)$ of equivalence classes of those $S$ systems $\left(S ;\left\{m_{\alpha}\right\}\right)$ of rank 1 satisfying $\left.\left.m_{1}=m, 1\right) \sim 8\right)$ and $\vartheta: S_{11} \cong F_{m}^{\nu}$, is in a bijective correspondence with the orbit space $\mathfrak{M}(m, \nu)$.

Proof. Let $\mathscr{S}=\left(S ;\left\{m_{\alpha}\right\}_{1 \leqq \alpha \leqq 2}\right)$ be an $S$-system of rank 1 satisfying $\left.m_{1}=m, 1\right) \sim 8$ ) and $S_{11} \cong \boldsymbol{F}_{m}^{\nu}$. We define

$$
(u, v)=\mathscr{R} e u v^{*} \quad \text { for } u, v \in S_{12} .
$$

Then 3) and 7) show that (, ) is an inner product on $S_{12}$ satisfying $(j u, j v)=(u, v)$ for $u, v \in S_{12}$. Put $W=\left(S_{12}, j\right)$ and

$$
\langle u, v\rangle=(u, v)+\sqrt{-1}(u, j v) \quad \text { for } u, v \in S_{12} .
$$


Then $\langle$,$\rangle is a hermitian inner product on W .1$ ), 5) and 8-1) show that $W$ is an $\boldsymbol{F}_{m}^{\nu}$-module over $\boldsymbol{C}$. Furthermore Remark 2 implies $(\lambda u, v)$ $=(u, \bar{\lambda} v)$ for $\lambda \in F_{m}^{\nu}, u, v \in S_{12}$, and hence

$$
\text { (*) }\langle\lambda u, v\rangle=\langle u, \bar{\lambda} v\rangle \quad \text { for } \lambda \in \boldsymbol{F}_{m}^{\nu}, u, v \in W .
$$

Now the correspondence $\mathscr{S} \leadsto W$ induces the map $\mathfrak{S}(m, \nu) \ni\{\mathscr{S}\} \mapsto[W]$ $\in \mathfrak{M}(m, \nu)$.

Conversely let $W$ be an $\boldsymbol{F}_{m}^{\nu}$-module with a hermitian inner product $\langle$,$\rangle satisfying the condition (*)$, and $W_{R}$ the scalar restriction to $\boldsymbol{R}$ of $W$. We define an inner product (, ) on $W_{R}$ by

$$
(u, v)=\mathscr{R} e\langle u, v\rangle \quad \text { for } u, v \in W .
$$

It satisfies

$$
(\lambda u, v)=(u, \bar{\lambda} v) \quad \text { for } \lambda \in \boldsymbol{F}_{m}^{\nu}, u, v \in W .
$$

The natural complex structure on $W_{R}$ (defined by the multiplication $\sqrt{-1}$ on $W$ ) will be denoted by $j$. Making use of the standard inner product $(\lambda, \mu)=\mathscr{R} e \lambda \bar{\mu}$ of $\boldsymbol{F}_{m}^{\nu}$, we define a bilinear map $W_{\boldsymbol{R}} \times W_{\boldsymbol{R}} \ni(u, v) \mapsto u v \in$ $\boldsymbol{F}_{m}^{\nu}$ by

$$
(u v, \lambda)=(u, \lambda v) \quad \text { for each } \lambda \in \boldsymbol{F}_{m}^{\nu} .
$$

It satisfies

$$
\begin{gathered}
\lambda(u v)=(\lambda u) v, \quad(u v) \lambda=u(\bar{\lambda} v), \\
\mathscr{R} e u v=(u, v), \\
\overline{u v}=v u, \\
(j u)(j v)=u v,
\end{gathered}
$$

for $u, v \in W$ and $\lambda \in \boldsymbol{F}_{m}^{\nu}$. Now we put

$$
S=\left\{\left(\begin{array}{ll}
\lambda & u \\
v & \xi
\end{array}\right) ; \begin{array}{l}
\lambda \in \boldsymbol{F}_{m}^{\nu}, \xi \in \boldsymbol{R} \\
u, v \in W_{\boldsymbol{R}}
\end{array}\right\}, \quad m_{1}=m, m_{2}=1
$$

and define on $S$ a product $s s^{\prime}$, an involution $s \mapsto s^{*}$ and a partial complex structure $j$ by

$$
\left(\begin{array}{ll}
\lambda & u \\
v & \xi
\end{array}\right)\left(\begin{array}{ll}
\lambda^{\prime} & u^{\prime} \\
v^{\prime} & \xi^{\prime}
\end{array}\right)=\left(\begin{array}{ll}
\lambda \lambda^{\prime}+u v^{\prime} & \lambda u^{\prime}+\xi^{\prime} u \\
\bar{\lambda}^{\prime} v+\xi v^{\prime} & \left(v, u^{\prime}\right)+\xi \xi^{\prime}
\end{array}\right),
$$




$$
\begin{aligned}
& \left(\begin{array}{ll}
\lambda & u \\
v & \xi
\end{array}\right)^{*}=\left(\begin{array}{ll}
\bar{\lambda} & v \\
u & \xi
\end{array}\right), \\
& j\left(\begin{array}{ll}
0 & u \\
v & 0
\end{array}\right)=\left(\begin{array}{lr}
0 & j u \\
j v & 0
\end{array}\right) .
\end{aligned}
$$

Then $\mathscr{S}=\left(S ;\left\{m_{\alpha}\right\}_{1 \leqq \alpha \leqq 2}\right)$ becomes an $S$-system of rank 1 with $S_{11}=F_{m}^{\nu}$. We define

$$
\begin{array}{ll}
\operatorname{tr} s=\mathscr{R} e \lambda+\xi & \text { for } s=\left(\begin{array}{ll}
\lambda & u \\
v & \xi
\end{array}\right) \in S, \\
\left(s, s^{\prime}\right)=\operatorname{tr} s s^{* *} & \text { for } s, s^{\prime} \in S,
\end{array}
$$

and hence

$$
(s, s)=(\lambda, \lambda)+(u, u)+(v, v)+\xi^{2} \quad \text { for } s=\left(\begin{array}{ll}
\lambda & u \\
v & \xi
\end{array}\right) \in S .
$$

We can show

$$
\begin{aligned}
\left(s, s^{\prime}\right) & =\left(s^{*}, s^{*}\right) & \text { for } s, s^{\prime} \in S, \\
\left(s s^{\prime}, s^{\prime \prime}\right) & =\left(s, s^{\prime \prime} s^{*}\right) & \text { for } s, s^{\prime}, s^{\prime \prime} \in S,
\end{aligned}
$$

making use of (3.1), (3.2) and (3.3). It follows from Remark 2 that $\mathscr{S}$ satisfies 2), 3) and 4). 1), 5), 6) and 8-1) follow from the definition. 7) and 8-2) follow from (3.5) and (3.2) respectively. Thus we get an $S$-system $\mathscr{S}$ of rank 1 satisfying $\left.\left.m_{1}=m, 1\right) \sim 8\right)$ and $S_{11}=F_{m}^{\nu}$. It is easy to see that the correspondence $W \sim \mathscr{S}$ induces the map $\mathfrak{M}(m, \nu)$ $\ni[W] \mapsto\{\mathscr{S}\} \in \widetilde{S}(m, \nu)$, and that it is the inverse of the former map $\mathfrak{S}(m, \nu) \rightarrow \mathfrak{M}(m, \nu)$. This proves Theorem 3.1. q.e.d.

Next we shall describe explicitly the hermitian map of the Siegel domain corresponding to $[W] \in \mathfrak{M}(m, \nu)$. The $\boldsymbol{C}$-linear extension to $\left(\boldsymbol{P}_{m}^{\nu}\right)^{\boldsymbol{C}}$ of the involutive linear automorphism $\lambda \mapsto \bar{\lambda}$ of $P_{m}^{\nu}$, will be also denoted by $\lambda \mapsto \bar{\lambda}$. Then $\left(X_{m}^{\nu}\right)^{c}$ is identified with the space

$$
H_{m}\left(\left(P_{m}^{\nu}\right)^{C}\right)=\left\{z \in M_{m}\left(\left(P_{m}^{\nu}\right)^{c}\right) ;{ }^{t} \bar{z}=z\right\}
$$

of all hermitian matrices in $M_{m}\left(\left(P_{m}^{\nu}\right)^{c}\right)$. The $(i, k)$-th component of $z \in$ $H_{m}\left(\left(P_{m}^{\nu}\right)^{c}\right)$ will be denoted by $z_{i k}$. Denoting the $C$-linear extension to $\left(P_{m}^{\nu}\right)^{c}$ of the standard inner product $($,$) on P_{m}^{\nu}$ by the same notation $($,$) , and the complex conjugation of \left(P_{m}^{\nu}\right)^{c}$ with respect to $P_{m}^{\nu}$ by $\lambda \mapsto \tilde{\lambda}$, we define a hermitian inner product $\langle$,$\rangle on \left(P_{m}^{\nu}\right)^{c}$ by 


$$
\langle\lambda, \mu\rangle=(\lambda, \tilde{\mu}) \quad \text { for } \lambda, \mu \in\left(P_{m}^{\nu}\right)^{c} .
$$

We define a map $H: W \times W \rightarrow\left(P_{m}^{\nu}\right)^{C}$ by

$$
\langle\lambda, H(u, v)\rangle=\langle\lambda v, u\rangle \quad \text { for each } \lambda \in P_{m}^{\nu} .
$$

It is sesqui-linear (i.e., $C$-linear with respect to $u$ and conjugate linear with respect to $v$ ) and satisfies

$$
\widetilde{H(u, v)}=\overline{H(v, u)} \quad \text { for } u, v \in W .
$$

In terms of the standard units $c_{0}, c_{1}, \cdots, c_{\nu-1}$ of $P_{m}^{\nu}, H$ is given by

$$
H(u, v)=\sum_{0 \leqq i \leqq \nu-1}\left\langle u, c_{i} v\right\rangle c_{i} \quad \text { for } u, v \in W .
$$

THEOREM 3.2. The $V_{m}^{\nu}$-positive hermitian map $F: Y \times Y \rightarrow H_{m}\left(\left(P_{m}^{\nu}\right)^{c}\right)$ of the Siegel domain $D\left(V_{m}^{\nu}, F\right)$ corresponding to $[W] \in \mathfrak{M}(m, \nu)$ is described as follows: Put $Y=\overbrace{W+\cdots+W}^{m}$. The $i$-th component of $u \in Y$ will be denoted by $u_{i} \in W(1 \leqq i \leqq m)$. Then $F$ is given by

$$
\begin{array}{ll}
F(u, v)_{i k}=\frac{1}{2}\left\{H\left(u_{i}, v_{k}\right)+\overline{\left.H\left(u_{k}, v_{i}\right)\right\}}\right. & \text { for } 1 \leqq i, k \leqq m, i \neq k, \\
F(u, v)_{i i}=\left\langle u_{i}, v_{i}\right\rangle & \text { for } 1 \leqq i \leqq m .
\end{array}
$$

Proof. Let $\mathscr{S}$ be an $S$-system constructed from $W$ as in Theorem 3.1, and $C$ an $S$-algebra constructed from $\mathscr{S}$ as in Theorem 2.1, (C). We may assume that the $T$-algebra $A$ associated to $C$ is $A_{m}^{\nu}$ and $Y=$ $\left\{a \in B ; a^{*}=a\right\}$ is identified with the totality of "matrices" of the form

$$
u=\left(\begin{array}{c|c}
0 & u_{1} \\
& u_{m} \\
\hline u_{1} \cdots u_{m} & 0
\end{array}\right] ; \quad u_{i} \in W_{\boldsymbol{R}}(1 \leqq i \leqq m),
$$

with the natural complex structure $j$. The associated symmetric map $\Phi$ is given by

$$
\begin{cases}\Phi(u, v)_{i k}=\frac{1}{2} \mathscr{P}\left(u_{i} v_{k}+v_{i} u_{k}\right) & \text { for } 1 \leqq i, k \leqq m, i \neq k \\ \Phi(u, v)_{i i}=\mathscr{R}_{e}\left(u_{i} v_{i}\right)=\left(u_{i}, v_{i}\right) & \text { for } 1 \leqq i \leqq m\end{cases}
$$

in the notation in Theorem 3.1, and hence

$$
\begin{cases}\Phi(u, j v)_{i k}=\frac{1}{2} \mathscr{P}\left(u_{i}\left(j v_{k}\right)-v_{i}\left(j u_{k}\right)\right) & \text { for } 1 \leqq i, k \leqq m, i \neq k \\ \Phi(u, j v)_{i i}=\left(u_{i}, j v_{i}\right) & \text { for } 1 \leqq i \leqq m\end{cases}
$$


We define an $R$-bilinear map $H: W_{\boldsymbol{R}} \times W_{\boldsymbol{R}} \rightarrow\left(P_{m}^{\nu}\right)^{\boldsymbol{C}}$ by

$$
H(u, v)=\mathscr{P}(u v+\sqrt{-1} u(j v)) \quad \text { for } u, v \in W,
$$

denoting the $C$-linear extension of $\mathscr{P}: \boldsymbol{F}_{m}^{\nu} \rightarrow P_{m}^{\nu}$ by the same $\mathscr{P}:\left(F_{m}^{\nu}\right)^{C} \rightarrow$ $\left(P_{m}^{\nu}\right)^{c}$. Then it is characterized by

$$
\langle\lambda, H(u, v)\rangle=\langle\lambda v, u\rangle \quad \text { for each } \lambda \in P_{m}^{\nu},
$$

and hence it coincides with the formerly defined sesqui-linear map $H$. In fact,

$$
\begin{aligned}
\langle\lambda, H(u, v)\rangle & =\langle\lambda, u v+\sqrt{-1} u(j v)\rangle=(\lambda, u v)-\sqrt{-1}(\lambda, u(j v)) \\
& =(\lambda, u v)+\sqrt{-1}(\lambda,(j u) v) \\
& =(\lambda v, u)+\sqrt{-1}(\lambda v, j u)=\langle\lambda v, u\rangle .
\end{aligned}
$$

Now, by (3.6), (3.6)', the associated hermitian map $F(u, v)=\frac{1}{2}\{\Phi(u, v)+$ $\sqrt{-1} \Phi(u, j v)\}$ is described as

$$
\begin{array}{ll}
2 F(u, v)_{i k}=\frac{1}{2}\left\{H\left(u_{i}, v_{k}\right)+\overline{H\left(u_{k}, v_{i}\right)}\right\} & \text { for } 1 \leqq i, k \leqq m, i \neq k, \\
2 F(u, v)_{i i}=\left\langle u_{i}, v_{i}\right\rangle & \text { for } 1 \leqq i \leqq m .
\end{array}
$$

This implies the Theorem.

q.e.d.

Remark 3. Let $D\left(V_{m}^{\nu}, F\right) \subset X^{c} \times Y$ be the Siegel domain constructed from $[W] \in \mathfrak{M}(m, \nu)$ in the Theorem. As we have seen in the proof of Theorem 2.2, Cor., we have the direct sum decomposition

$$
\operatorname{gl}\left(D\left(V_{m}^{\nu}, F\right)\right)=\mathfrak{g}_{0} \oplus \mathfrak{g}^{c}
$$

where $g_{0}$ is the kernel of the projection $\pi_{X}$. The ideal $g_{0}$ is described as follows: Let $\mathfrak{S}_{0}$ denote the real subalgebra of $\operatorname{gl}(W)$ consisting of those linear endomorphisms of $W$, leaving invariant the hermitian inner product $\langle$,$\rangle of W$, and commuting with the action of $\boldsymbol{F}_{m}^{\nu}$ on $W$. We define $\psi_{D} \in \mathfrak{g l}\left(X^{C} \times Y\right)$ for $D \in \mathfrak{D}_{0}$ by

$$
\psi_{D}\left(z, \sum_{i} u_{i}\right)=\left(0, \sum_{i} D u_{i}\right) \quad \text { for } z \in X^{c}, u_{i} \in W(1 \leqq i \leqq m) .
$$

Then the correspondence $D \mapsto \psi_{D}$ gives an isomorphism of $\mathfrak{D}_{0}$ onto $g_{0}$. This follows from the Theorem or from the detailed description of $g_{0}$ in terms of derivations of $C$, given in Takeuchi [7]. The subalgebra $g^{c}$ is also described explicitly by means of the $\boldsymbol{F}_{m}^{\nu}$-module structure of $W$ [7].

It is easy to determine $\mathfrak{M}(m, \nu)$, since the set $\mathfrak{M}\left(\boldsymbol{F}_{m}^{\nu}\right)$ is well known. 
In the following we will give $\mathfrak{M}(m, \nu)$ for each $(m, \nu)$.

Case $m=2, \nu \geqq 1$.

$F_{m}^{\nu}=C_{\nu-1}$. Note that the action of each $\sigma \in O(\nu-1)$ on $R^{\nu-1}=\left(C_{\nu-1}\right)_{1}$ is uniquely extended to an automorphism $\tilde{\sigma}$ of $C_{\nu-1}$, and that $\operatorname{Aut}^{*}\left(\boldsymbol{F}_{m}^{\nu}, \mathscr{P}\right)$ coincides with the totality of $\tilde{\sigma}$ for $\sigma \in O(\nu-1)$.

(a) $\nu=2 \ell+1(\ell \geqq 0)$.

$$
\mathfrak{M}(m, \nu)=\left\{\left[W_{r}\right] ; r \in Z, r \geqq 0\right\}, \quad \operatorname{dim} W_{r}=2^{\ell} r
$$

(b) $\nu=2 \ell+2(\ell \geqq 0)$.

$$
\mathfrak{M}(m, \nu)=\left\{\left[W_{r, s}\right] ; r, s \in Z, r \geqq s \geqq 0\right\}, \quad \operatorname{dim} W_{r, s}=2^{\ell}(r+s) .
$$

$W_{r, s}=\underbrace{W^{(1)} \oplus \ldots \oplus W^{(1)}}_{r} \oplus \underbrace{W^{(2)} \oplus \cdots \oplus W^{(2)}}_{s}$, where $W^{(1)}$ and $W^{(2)}$ are inequivalent irreducible $C_{2 \ell+1}$-modules over $C$.

Case $m \neq 2, \nu=1,2,4$ or 8 .

(a) $\nu=1(m \neq 2) . \quad \boldsymbol{F}_{m}^{\nu}=\boldsymbol{R}$ is isomorphic to $C_{0}$ as an algebra with involution and projection $\mathscr{P}$.

$$
\mathfrak{M}(m, \nu)=\left\{\left[W_{r}\right] ; r \in Z, r \geqq 0\right\}, \quad \operatorname{dim} W_{r}=r .
$$

(b) $\nu=2(m \geqq 3) . \quad F_{m}^{\nu}=C$ (as a real algebra) is isomorphic to $C_{1}$ as an algebra with involution and $\mathscr{P}$.

$$
\mathfrak{M}(m, \nu)=\left\{\left[W_{r, s}\right] ; r, s \in Z, r \geqq s \geqq 0\right\}, \quad \operatorname{dim} W_{r, s}=r+s .
$$

$W_{r, s}=\underbrace{W^{(1)} \oplus \ldots \oplus W^{(1)}}_{r} \oplus \underbrace{W^{(2)} \oplus \ldots \oplus W^{(2)}}_{s}$, where $W^{(1)}$ and $W^{(2)}$ are inequivalent irreducible $C_{1}$-modules over $\boldsymbol{C}$.

(c) $\nu=4(m \geqq 3) . \quad \boldsymbol{F}_{m}^{\nu}=\boldsymbol{H}$ (real quaternion algebra) is isomorphic to $C_{2}$ as an involutive algebra.

$$
\mathfrak{M}(m, \nu)=\left\{\left[W_{r}\right] ; r \in Z, r \geqq 0\right\}, \quad \operatorname{dim} W_{r}=2 r .
$$

(d) $\nu=8(m=3) . \quad \boldsymbol{F}_{m}^{\nu}=\boldsymbol{K}$ (real Cayley algebra).

$$
\mathfrak{M}(m, \nu)=\left\{\left[W_{0}\right]\right\}, \quad \operatorname{dim} W_{0}=0 .
$$

\section{Symmetric Siegel domains}

Let $C$ be an $S$-algebra corresponding to $[W] \in \mathfrak{M}(m, \nu)$ as in the previous section, and $F:(Y, j) \times(Y, j) \rightarrow X^{c}, X=X_{m}^{\nu}$, be the $V_{m}^{\nu}$-positive 
hermitian map associated to $C$. We want to describe the Satake's third condition (iii) for $D\left(V_{m}^{\nu}, F\right)$ in terms of the $S$-algebra $C$. The relations cited in Lemma of $\S 2$ will be used consistently throughout the following computations. Recall

$$
\begin{array}{ll}
F(u, v)=\frac{1}{4}\{(\hat{u} v+\hat{v} u)+\sqrt{-1}(\hat{u}(j v)+(j \hat{v}) u)\} & \text { for } u, v \in Y, \\
R(x) u=\frac{1}{2} \psi^{\prime \prime}(x) u=\frac{1}{2}(x u+u x)=\frac{1}{2}\left(x \hat{u}+v_{v}\right) & \text { for } x \in X, u \in Y,
\end{array}
$$

and hence

$$
\begin{gathered}
\widehat{R(x) u}=\frac{1}{2} x \hat{u}, \quad R(x) u=\frac{1}{2} u x \quad \text { for } x \in X, u \in Y, \\
R(x+\sqrt{-1} y) u=\frac{1}{2}\{(x \hat{u}+\underset{v}{x})+j(y \hat{u}+u y)\} \quad \text { for } x, y \in X, u \in Y .
\end{gathered}
$$

We have

$$
\begin{aligned}
& 16 R(F(u, v)) R(x) u=(\hat{u} \underline{v}+\hat{v} \underline{u})(x \hat{u})+(\underline{u} x)(\hat{u} v+\hat{v} u) \\
& +j\{(\hat{v}(j v)+(j \hat{v}) \underset{v}{u})(x \hat{u})+(\underset{v}{u})(\hat{u}(j v)+(j \hat{v}) u)\}, \\
& 8 F(u, R(x) v)=\hat{u}(v x)+(x \hat{v}) u+\sqrt{-1}\{\hat{u}(j(v x))+(j(x \hat{v})) u\}, \\
& 16 R(F(u, R(x) v)) u=(\hat{u}(v x)+(x \hat{v}) \underset{v}{u}) \hat{u}+\underline{u}(\hat{u}(v x)+(x \hat{v}) u) \\
& +j\{[\hat{u}(j(v x))+(j(x \hat{v})) u] \hat{u}+u[\hat{u}(j(v x))+(j(x \hat{v})) u]\},
\end{aligned}
$$

and hence the condition (iii) is equivalent to

$$
\begin{aligned}
& (\hat{u} v+\hat{v} u)(x \hat{u})+j\{(\hat{u}(j v)+(j \hat{v}) u)(x \hat{u})\} \\
& =(\hat{u}(v x)+(x \hat{v}) u) \hat{u}+j\{[\hat{u}(j(v x))+(j(x \hat{v})) u] \hat{u}\} \\
& \qquad \\
& \text { for each } u, v \in Y, x \in X .
\end{aligned}
$$

Comparing the $C_{i, m+1}$-components, we get the equivalent conditions

$$
\begin{aligned}
\sum_{1 \leqq k, \ell \leqq m}\left\{\left(u_{i, m+1} v_{m+1, k}\right)\left(x_{k \ell} u_{\ell, m+1}\right)+\left(v_{i, m+1} u_{m+1, k}\right)\left(x_{k \ell} u_{\ell, m+1}\right)\right. \\
\left.\quad+\left(u_{i, m+1}\left(j v_{m+1, k}\right)\right)\left(x_{k \ell}\left(j u_{\ell, m+1}\right)\right)+\left(\left(j v_{i, m+1}\right) u_{m+1, k}\right)\left(x_{k \ell}\left(j u_{\ell, m+1}\right)\right)\right\} \\
=\sum_{1 \leqq k, \ell \leqq m}\left\{\left(u_{i, m+1}\left(v_{m+1, k} x_{k \ell}\right)\right) u_{\ell, m+1}+\left(\left(x_{i k} v_{k, m+1}\right) u_{m+1, \ell}\right) u_{\ell, m+1}\right. \\
\left.\quad+\left[u_{i, m+1}\left(\left(j v_{m+1, k}\right) x_{k \ell}\right)\right] j u_{\ell, m+1}+\left[\left(x_{i k}\left(j v_{k, m+1}\right)\right) u_{m+1, \ell}\right] j u_{\ell, m+1}\right\} \\
\quad \quad \text { for each } 1 \leqq i \leqq m, u=\left(u_{k \ell}\right), v=\left(v_{k \ell}\right) \in Y, x=\left(x_{k \ell}\right) \in X .
\end{aligned}
$$

In the following, the general element of $C_{i k}(1 \leqq i, k \leqq m)$ and $C_{i, m+1}$ $\left(1 \leqq i \leqq m\right.$ ) will be denoted by $x_{i k}$ and $u_{i}, v_{i}, w_{i}, \cdots$ respectively. If we write the above conditions for 


$$
\begin{aligned}
& x=x_{k \ell}+x_{k \ell}^{*}, \quad k \neq i, \quad \ell \neq i, \\
& x=x_{i k}+x_{i k}^{*}, \quad k \neq i, \\
& x=x_{i i}, \quad x_{i i}^{*}=x_{i i},
\end{aligned}
$$

we have the following three conditions:

$$
\begin{aligned}
\left(u_{i} v_{k}^{*}\right)\left(x_{k \ell} u_{\ell}\right)+\left(u_{i} v_{\ell}^{*}\right)\left(x_{k \ell}^{*} u_{k}\right)+\left(v_{i} u_{k}^{*}\right)\left(x_{k \ell} u_{\ell}\right)+\left(v_{i} u_{\ell}^{*}\right)\left(x_{k \ell}^{*} u_{k}\right) & \\
& +\left(u_{i} j v_{k}^{*}\right)\left(x_{k \ell} j u_{\ell}\right)+\left(u_{i} j v_{\ell}^{*}\right)\left(x_{k \ell}^{*} j u_{k}\right)+\left(\left(j v_{i}\right) u_{k}^{*}\right)\left(x_{k \ell} j u_{\ell}\right) \\
& +\left(\left(j v_{i}\right) u_{\ell}^{*}\right)\left(x_{k \ell}^{*} j u_{k}\right) \\
= & \left(u_{i}\left(v_{k}^{*} x_{k \ell}\right)\right) u_{\ell}+\left(u_{i}\left(v_{\ell}^{*} x_{k \ell}^{*}\right)\right) u_{k}+\left[u_{i}\left(\left(j v_{k}^{*}\right) x_{k \ell}\right)\right] j u_{\ell} \\
& +\left[u_{i}\left(\left(j v_{\ell}^{*}\right) x_{k \ell}^{*}\right)\right] j u_{k} \quad \text { if } k \neq i, \ell \neq i
\end{aligned}
$$

(iii-2) $\left(u_{i} v_{i}^{*}\right)\left(x_{i k} u_{k}\right)+\left(u_{i} v_{k}^{*}\right)\left(x_{i k}^{*} u_{i}\right)+\left(v_{i} u_{i}^{*}\right)\left(x_{i k} u_{k}\right)+\left(v_{i} u_{k}^{*}\right)\left(x_{i k}^{*} u_{i}\right)$

$$
\begin{aligned}
& +\left(u_{i} j v_{i}^{*}\right)\left(x_{i k} j u_{k}\right)+\left(u_{i} j v_{k}^{*}\right)\left(x_{i k}^{*} u_{i}\right)+\left(\left(j v_{i}\right) u_{i}^{*}\right)\left(x_{i k} j u_{k}\right) \\
& +\left(\left(j v_{i}\right) u_{k}^{*}\right)\left(x_{i k}^{*} j u_{i}\right) \\
= & \left(u_{i}\left(v_{i}^{*} x_{i k}\right)\right) u_{k}+\left(u_{i}\left(v_{k}^{*} x_{i k}^{*}\right)\right) u_{i}+\sum_{\substack{1 \leq \ell \leq m \\
\ell \neq i}}\left(\left(x_{i k} v_{k}\right) u_{\ell}^{*}\right) u_{\ell} \\
& +\left(\left(x_{i k} v_{k}\right) u_{i}^{*}\right) u_{i}+\left[u_{i}\left(\left(j v_{i}^{*}\right) x_{i k}\right)\right] j u_{k}+\left[u_{i}\left(\left(j v_{k}^{*}\right) x_{i k}^{*}\right)\right] j u_{i} \\
& +\sum_{\substack{1 \leq \ell \leq m \\
\ell \neq i}}\left(\left(x_{i k} j v_{k}\right) u_{\ell}^{*}\right) j u_{\ell}+\left[\left(x_{i k}\left(j v_{k}\right)\right) u_{i}^{*}\right] j u_{i} \quad \text { if } k \neq i .
\end{aligned}
$$

(iii-3) $\left(u_{i} v_{i}^{*}\right)\left(x_{i i} u_{i}\right)+\left(v_{i} u_{i}^{*}\right)\left(x_{i i} u_{i}\right)+\left(u_{i} j v_{i}^{*}\right)\left(x_{i i} j u_{i}\right)+\left(\left(j v_{i}\right) u_{i}^{*}\right)\left(x_{i i} u_{i}\right)$

$$
\begin{aligned}
= & \left(u_{i}\left(v_{i}^{*} x_{i i}\right)\right) u_{i}+\sum_{1 \leqq k \leqq m}\left(\left(x_{i i} v_{i}\right) u_{k}^{*}\right) u_{k}+\left(u_{i}\left(j v_{i}^{*} x_{i i}\right)\right) j u_{i} \\
& +\sum_{1 \leqq k \leqq m}\left(\left(x_{i i} j v_{i}\right) u_{k}^{*}\right) j u_{k} \quad \text { for each } 1 \leqq i \leqq m .
\end{aligned}
$$

Thus the condition (iii) is equivalent to the conditions (iii-1), (iii-2), (iii-3) as a whole. In the equality (iii-1), $\left(u_{i} v_{k}^{*}\right)\left(x_{k \ell} u_{\ell}\right)=\left(\left(u_{i} v_{k}^{*}\right) x_{k \ell}\right) u_{\ell}=$ $\left(u_{i}\left(v_{k}^{*} x_{k \ell}\right)\right) u_{\ell}$. In the same way, $\left(u_{i} v_{\ell}^{*}\right)\left(x_{k \ell}^{*} u_{k}\right)=\left(u_{i}\left(v_{\ell}^{*} x_{k \ell}^{*}\right)\right) u_{k},\left(u_{i} j v_{k}^{*}\right)\left(x_{k \ell} j u_{\ell}\right)$ $=\left[u_{i}\left(\left(j v_{k}^{*}\right) x_{k \ell}\right)\right] j u_{\ell} \quad$ and $\quad\left(u_{i} j v_{\ell}^{*}\right)\left(x_{k \ell}^{*} j u_{k}\right)=\left[u_{i}\left(\left(j v_{\ell}^{*}\right) x_{k \ell}^{*}\right)\right] j u_{\ell} . \quad$ Furthermore $\left(v_{i} u_{\ell}^{*}\right)\left(x_{k \ell}^{*} u_{k}\right)=\left(\left(v_{i} u_{\ell}^{*}\right) x_{k \ell}^{*}\right) u_{k}=\left(v_{i}\left(u_{\ell}^{*} x_{k \ell}^{*}\right)\right) u_{k}=\left(v_{i}\left(x_{k \ell} u_{\ell}\right) *\right) u_{k}$. In the same way, $\left(\left(j v_{i}\right) u_{\ell}^{*}\right)\left(x_{k \ell}^{*} j u_{k}\right)=\left(j v_{i}\left(x_{k \ell} u_{\ell}\right)^{*}\right) j u_{k}$. Thus (iii-1) is equivalent to

$$
\begin{aligned}
(\mathrm{iii}-1)^{\prime} \quad\left(v_{i} u_{k}^{*}\right)\left(x_{k \ell} u_{\ell}\right)+\left(v_{i}\left(x_{k \ell} u_{\ell}\right) *\right) u_{k}+\left(\left(j v_{i}\right) u_{k}^{*}\right) j\left(x_{k \ell} u_{\ell}\right) \\
+\left(\left(j v_{i}\right)\left(x_{k \ell} u_{\ell}\right) * j u_{k}=0 \quad \text { if } k \neq i, \ell \neq i .\right.
\end{aligned}
$$

In the lefthand side of the equality (iii-2), $\left(u_{i} v_{i}^{*}\right)\left(x_{i k} u_{k}\right)+\left(v_{i} u_{i}^{*}\right)\left(x_{i k} u_{k}\right)$ $=\left(\left(u_{i} v_{i}^{*}\right) x_{i k}\right) u_{k}+\left(\left(v_{i} u_{i}^{*}\right) x_{i k}\right) u_{k}=\left(u_{i}\left(v_{i}^{*} x_{i k}\right)\right) u_{k}+\left(v_{i}\left(u_{i}^{*} x_{i k}\right)\right) u_{k} . \quad$ In the same way, $\left(u_{i} j v_{i}^{*}\right)\left(x_{i k} j u_{k}\right)+\left(\left(j v_{i}\right) u_{i}^{*}\right)\left(x_{i k} j u_{k}\right)=\left[u_{i}\left(\left(j v_{i}^{*}\right) x_{i k}\right)\right] j u_{k}+\left[\left(j v_{i}\right)\left(u_{i}^{*} x_{i k}\right)\right] j u_{k}$. In the righthand side of (iii-2), $\left(u_{i}\left(v_{k}^{*} x_{i k}^{*}\right)\right) u_{i}+\left(\left(x_{i k} v_{k}\right) u_{i}^{*}\right) u_{i}=\left(\left(u_{i} v_{k}^{*}\right) x_{i k}^{*}\right) u_{i}$ 
$+\left(x_{i k}\left(v_{k} u_{i}^{*}\right)\right) u_{i}=\left(u_{i} v_{k}^{*}\right)\left(x_{i k}^{*} u_{i}\right)+x_{i k}\left(\left(v_{k} u_{i}^{*}\right) u_{i}\right)$. In the same way, [ $u_{i}\left(\left(j v_{k}^{*}\right)\right.$ $\left.\left.\times x_{i k}^{*}\right)\right] j u_{i}+\left(\left(x_{i k} j v_{k}\right) u_{i}^{*}\right) j u_{i}=\left(u_{i} j v_{k}^{*}\right)\left(x_{i k}^{*} j u_{i}\right)+x_{i k}\left[\left(\left(j v_{k}\right) u_{i}^{*}\right) j u_{i}\right]$. Thus (iii-2) is equivalent to

$$
\begin{array}{rlr}
(\mathrm{iii}-2)^{\prime} & \left(v_{i}\left(x_{i k}^{*} u_{i}\right)^{*}\right) u_{k}+\left(v_{i} u_{k}^{*}\right)\left(x_{i k}^{*} u_{i}\right)+\left(\left(j v_{i}\right)\left(x_{i k}^{*} u_{i}\right)^{*}\right) j u_{k}+\left(\left(j v_{i}\right) u_{k}^{*}\right) j\left(x_{i k}^{*} u_{i}\right) \\
= & x_{i k}\left\{\left(v_{k} u_{i}^{*}\right) u_{i}+\left(\left(j v_{k}\right) u_{i}^{*}\right) j u_{i}\right\} & \text { if } i \neq k . \\
& +\sum_{\substack{1 \leq \ell \leq m \\
\ell \neq i}}\left\{\left(\left(x_{i k} v_{k}\right) u_{\ell}^{*}\right) u_{\ell}+\left[\left(j\left(x_{i k} v_{k}\right)\right) u_{\ell}^{*}\right] j u_{\ell}\right\} &
\end{array}
$$

Furthermore (iii-3) is equivalent to

$(\mathrm{iii}-3)^{\prime} \quad \sum_{\substack{1 \leqq k \leqq m \\ k \neq i}}\left\{\left(v_{i} u_{k}^{*}\right) u_{k}+\left(\left(j v_{i}\right) u_{k}^{*}\right) j u_{k}\right\}=0 \quad$ for each $1 \leqq i \leqq m$.

Now, if $m=1$, the conditions (iii-1)', (iii-2)', (iii-3)' are always satisfied. If $m \geqq 2$, the condition (iii-1) for $k=\ell \neq i$ implies

$$
\left(v_{i} u_{k}^{*}\right) u_{k}+\left(\left(j v_{i}\right) u_{k}^{*}\right) j u_{k}=0 \quad \text { if } i \neq k,
$$

or equivalently

$(4.1)^{\prime} \quad\left(v_{i} u_{k}^{*}\right) w_{k}+\left(v_{i} w_{k}^{*}\right) u_{k}+\left(\left(j v_{i}\right) u_{k}^{*}\right) j w_{k}+\left(\left(j v_{i}\right) w_{k}^{*}\right) j u_{k}=0 \quad$ if $i \neq k$.

Actually the condition (4.1) is equivalent to three conditions (iii-1)', (iii-2)', (iii-3)' . In fact; assume (4.1), then replacing $w_{k}=x_{k \ell} u_{\ell}$ in (4.1)', we get (iii-1)'. Replacing $u_{k}=x_{i k}^{*} u_{i}$ and $w_{k}=u_{k}$ in $(4.1)^{\prime}$, we know that the lefthand side of (iii-2) ${ }^{\prime}$ is 0 . The righthand side of (iii-2) ${ }^{\prime}$ is also 0 by (4.1). (iii-3)' follows from (4.1). These prove the required assertion.

Now we are in a position to prove Theorem B in Introduction.

Proof of Theorem $B$. Passing to the $\boldsymbol{F}_{m}^{\nu}$-module $W$, the condition (4.1) is equivalent to

$$
(\mathscr{P}(v u)) u+[\mathscr{P}((j v) u)] j u=0 \quad \text { for } u, v \in W_{\boldsymbol{R}} \text {. }
$$

Recalling that $(\mathscr{P}(u v), \lambda)=(u v, \lambda)=(u, \lambda v)$ for $\lambda \in P_{m}^{\nu}$, we get

$$
\mathscr{P}(u v)=\sum_{0 \leqq i \leqq \nu-1}\left(u, c_{i} v\right) c_{i} \quad \text { for } u, v \in W_{\boldsymbol{R}} .
$$

Thus (4.2) is equivalent to

$$
\sum_{0 \leqq i \leqq \nu-1}\left\{\left(v, c_{i} u\right)\left(c_{i} u, w\right)-\left(j v, c_{i} u\right)\left(c_{i} u, j w\right)\right\}=0 \quad \text { for } u, v, w \in W_{R},
$$


or equivalently

$$
\sum_{0 \leqq i \leqq \nu-1}\left\{\left(v, c_{i} u\right)\left(c_{i} u, j w\right)+\left(j v, c_{i} u\right)\left(c_{i} u, w\right)\right\}=0 \quad \text { for } u, v, w \in W_{\boldsymbol{R}} .
$$

Thus the condition (4.2) is equivalent to

$$
\sum_{0 \leqq i \leqq \nu-1}\left\langle c_{i} u, v\right\rangle\left\langle c_{i} u, w\right\rangle=0 \quad \text { for } u, v, w \in W .
$$

This proves Theorem B.

q.e.d.

Let $\mathfrak{M}_{s}(m, \nu)$ denote the set of those $[W] \in \mathfrak{M}(m, \nu)$ defining symmetric Siegel domains.

Lemma. Let $[W] \in \mathfrak{M}_{s}(m, \nu), N=\operatorname{dim} W$ and $\rho: \boldsymbol{F}_{m}^{\nu} \rightarrow$ End $W$ be the representation defined by the $\boldsymbol{F}_{m}^{\nu}$-module structure on $W$.

1) If $m \geqq 2$, then

$$
N(N-\nu+2)=\sum_{k=1}^{\nu-1}\left|\operatorname{Tr} \rho\left(c_{k}\right)\right|^{2} .
$$

2) If $\nu \geqq 3$, then $\operatorname{Tr} \rho\left(c_{k}\right)=0$ for each $1 \leqq k \leqq \nu-1$.

3) If $m \geqq 2$ and $\nu \neq 2$, then $N=0$ or $N=\nu-2$.

4) If $m \geqq 2$ and $\nu=2$, then $[W]=\left[W_{N, 0}\right]$, i.e., $W$ is the direct sum of $N$-copies of an irreducible $C_{1}$-module over $C$.

Proof. 1) Let $m \geqq 2$. Take an orthonormal basis $\left\{u_{1}, \cdots, u_{N}\right\}$ of $W$, and put

$$
a_{\alpha \beta k}=\left\langle c_{k} u_{\beta}, u_{\alpha}\right\rangle \quad \text { for } 1 \leqq k \leqq \nu-1,1 \leqq \alpha, \beta \leqq N .
$$

Then $\bar{a}_{\alpha \beta k}=-a_{\beta \alpha k}$ and $\rho\left(c_{k}\right)(1 \leqq k \leqq \nu-1)$ has the matricial representation $\left(a_{\alpha \beta k}\right)_{1 \leqq \alpha, \beta \leqq N}$. From the polarized form

$$
\sum_{i=0}^{\nu-1}\left\{\left\langle c_{i} u, v\right\rangle\left\langle c_{i} u^{\prime}, w\right\rangle+\left\langle c_{i} u^{\prime}, v\right\rangle\left\langle c_{i} u, w\right\rangle\right\}=0 \quad \text { for } u, u^{\prime}, v, w \in W
$$

of the condition $(\sharp)$, we have

$$
\delta_{\alpha \beta} \delta_{\gamma \delta}+\delta_{\gamma \beta} \delta_{\alpha \delta}+\sum_{k=1}^{\nu-1}\left(a_{\beta \alpha k} a_{\delta_{\gamma} k}+a_{\beta \gamma k} a_{\delta \alpha k}\right)=0
$$

for each $1 \leqq \alpha, \beta, \gamma, \delta \leqq N$. Putting $\beta=\alpha$ and $\delta=\gamma$, we get

$$
1+\delta_{\alpha \gamma}+\sum_{k}\left(a_{\alpha \alpha k} a_{\gamma \gamma k}+a_{\alpha \gamma k} a_{\gamma \alpha k}\right)=0
$$


Taking the sum over $\alpha$ and $\gamma$, we get

$$
N^{2}+N+\sum_{k}\left(\operatorname{Tr} \rho\left(c_{k}\right)\right)^{2}+\sum_{k} \operatorname{Tr}\left(\rho\left(c_{k}\right)^{2}\right)=0 .
$$

Since $\rho\left(c_{k}\right)^{2}=\rho\left(c_{k}^{2}\right)=-1_{W}$ and hence $\operatorname{Tr}\left(\rho\left(c_{k}\right)^{2}\right)=-N$, we have

$$
N(N+1)+\sum_{k}\left(\operatorname{Tr} \rho\left(c_{k}\right)\right)^{2}-(\nu-1) N=0 .
$$

Hence

$$
N(N-\nu+2)=-\sum_{k}\left(\operatorname{Tr} \rho\left(c_{k}\right)\right)^{2}=\sum_{k}\left|\operatorname{Tr} \rho\left(c_{k}\right)\right|^{2} .
$$

2) We may asume $F_{m}^{\nu} \neq K$, since $K$ has no modules other than the trivial one. Let $\left(\boldsymbol{F}_{m}^{\nu}\right)^{0}$ denote the orthogonal complement in $\boldsymbol{F}_{m}^{\nu}$ of the center of $\boldsymbol{F}_{m}^{\nu}$, with respect to the standard inner product on $\boldsymbol{F}_{m}^{\nu}$. Then $\left(F_{m}^{\nu}\right)^{0}$ is closed under the bracket product $[\lambda, \mu]=\lambda \mu-\mu \lambda$ and coincides with the commutator subalgebra $\left[\left(\boldsymbol{F}_{m}^{\nu}\right)^{0},\left(\boldsymbol{F}_{m}^{\nu}\right)^{0}\right]$. The assertion follows from the fact that each $c_{k}(1 \leqq k \leqq \nu-1)$ belongs to $\left(F_{m}^{\nu}\right)^{0}$ for $\nu \geqq 3$.

$3)$ is an immediate consequence of 1) and 2).

4) As we have seen in $\S 3$, if $m \geqq 2$ and $\nu=2$, then $\mathfrak{M}(m, \nu)=$

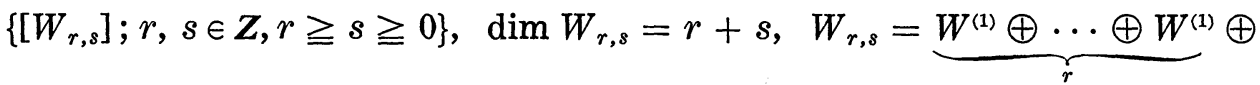
$\underbrace{W^{(2)} \oplus \cdots \oplus W^{(2)}}_{s}$, where $W^{(1)}$ and $W^{(2)}$ are inequivalent irreducible $C_{1^{-}}$ modules over $C$. Let $W=W_{r, s}$ and $[W] \in \mathfrak{M}_{s}(m, \nu)$. We may assume that $\rho\left(c_{1}\right)$ has the matricial representation

$$
\left(\begin{array}{llll}
\sqrt{-1} & 1_{r} & & \\
& & -\sqrt{-1} & 1_{s}
\end{array}\right),
$$

and hence $\left|\operatorname{Tr} \rho\left(c_{1}\right)\right|^{2}=(r-s)^{2}$. Now the assertion 1) implies

$$
(r+s)^{2}=(r-s)^{2},
$$

and hence $r \geqq s=0$.

The set $\mathfrak{M}_{s}(m, \nu)$ can be determined by the above Lemma. For example, let $m=2, \nu=2 \ell+2(\ell \geqq 1)$. Recall that $F_{m}^{\nu}=C_{2 \ell+1}$ and

$$
\mathfrak{M}(m, \nu)=\left\{\left[W_{r, s}\right] ; r, s \in Z, r \geqq s \geqq 0\right\}, \operatorname{dim} W_{r, s}=2^{\ell}(r+s) .
$$

If $\left[W_{r, s}\right] \in \mathfrak{M}_{s}(m, \nu)$, from Lemma 3$)$ it follows 


$$
r+s=0 \text { or } 2^{\ell}(r+s)=2 \ell,
$$

and hence the possibilities are one of

1) $\ell \geqq 1, r=s=0$,

2) $\ell=1, r=1, s=0\left(\nu=4, \operatorname{dim} W_{1,0}=2\right)$,

3) $\ell=2, r=1, s=0\left(\nu=6, \operatorname{dim} W_{1,0}=4\right)$.

We shall show that for each of the above $(r, s), W=W_{r, s}$ satisfies the condition (\#).

Case 1). The condition (\#) is clearly satisfied, since $W=\{0\}$.

Case 2). Note first that in terms of the sesqui-linear map $H$, the condition (\#) is written equivalently as

$$
\langle H(u, v) u, w\rangle=2\langle u, v\rangle\langle u, w\rangle \quad \text { for } u, v, w \in W .
$$

Let $W=C^{2}$ with the hermitian inner product $\langle u, v\rangle={ }^{t} u \bar{v}$. The $\boldsymbol{R}$ linear map $\rho: P_{m}^{\nu} \rightarrow M_{2}(C)$ defined by

$$
\begin{array}{rlr}
\rho\left(c_{0}\right)=\left(\begin{array}{ll}
1 & 0 \\
0 & 1
\end{array}\right), & \rho\left(c_{1}\right)=\left(\begin{array}{cc}
\sqrt{-1} & 0 \\
0 & -\sqrt{-1}
\end{array}\right), \\
\rho\left(c_{2}\right)=\left(\begin{array}{cc}
0 & -1 \\
1 & 0
\end{array}\right), & \rho\left(c_{3}\right)=\left(\begin{array}{cc}
0 & -\sqrt{-1} \\
-\sqrt{-1} & 0
\end{array}\right)
\end{array}
$$

gives rise to an irreducible $\boldsymbol{F}_{m}^{\nu}$-module structure on $W$ satisfying $\langle\lambda u, v\rangle$ $=\langle u, \bar{\lambda} v\rangle$. Through the injective map $\rho$, we identify $P_{m}^{\nu}$ with a real subspace of $M_{2}(C)$, and thus $\left(P_{m}^{\nu}\right)^{C}$ with $M_{2}(C)$. Then the hermitian inner product $\langle$,$\rangle on \left(P_{m}^{\nu}\right)^{C}$ is given by

$$
\langle\lambda, \mu\rangle=\frac{1}{2} \operatorname{Tr}\left(\lambda^{t} \bar{\mu}\right) \quad \text { for } \lambda, \mu \in M_{2}(C) .
$$

Now the sesqui-linear map $H: C^{2} \times C^{2} \rightarrow M_{2}(C)$ is given by

$$
H(u, v)=2 u^{t} \bar{v} \quad \text { for } u, v \in C^{2} .
$$

In fact, $\left\langle\lambda, 2 u^{t} \bar{v}\right\rangle=\operatorname{Tr} \lambda\left(v^{t} \bar{u}\right)=\operatorname{Tr}(\lambda v)^{t} \bar{u}=\langle\lambda v, u\rangle$ for each $\lambda \in M_{2}(C)$. Hence we have

$$
\begin{aligned}
\langle H(u, v) u, w\rangle & =\left\langle 2\left(u^{t} \bar{v}\right) u, w\right\rangle=2\left\langle u\left({ }^{t} \bar{v} u\right), w\right\rangle \\
& =2\langle u, v\rangle\langle u, w\rangle \text { for } u, v, w \in C^{2} .
\end{aligned}
$$

This shows the condition (\#) for $W$.

Case 3). Let $W=C^{4}$ with the hermitian inner product $\langle u, v\rangle={ }^{t} u \bar{v}$. The homomorphism $\rho: F_{m}^{\nu} \rightarrow M_{4}(C)$ defined by 


$$
\begin{aligned}
& \rho\left(c_{0}\right)=\left(\begin{array}{ll|ll}
1 & 0 & 0 \\
0 & 1 & & \\
\hline & 0 & 1 & 0
\end{array}\right), \quad \rho\left(c_{1}\right)=\left(\begin{array}{cc|c}
\sqrt{-1} & 0 & 0 \\
0 & \sqrt{-1} & 0 \\
\hline 0 & 1
\end{array}\right),
\end{aligned}
$$

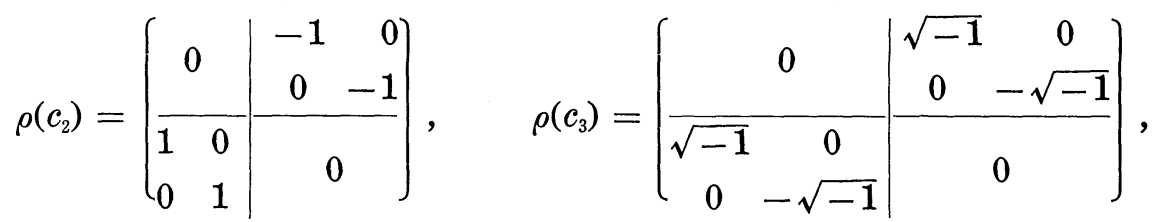

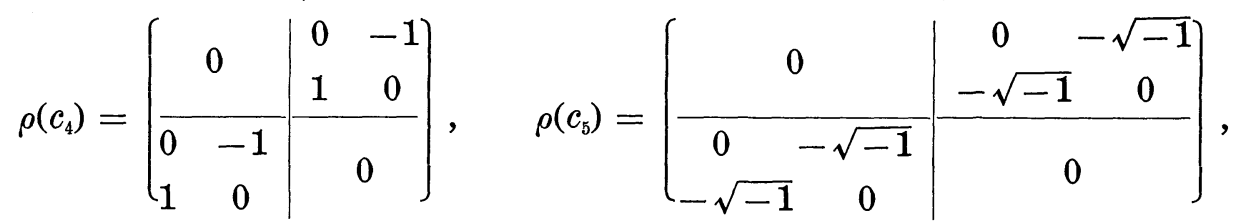

gives rise to an irreducible $\boldsymbol{F}_{m}^{\nu}$-module structure on $W$ satisfying $\langle\lambda u, v\rangle$ $=\langle u, \bar{\lambda} v\rangle$. It can be checked that the $\boldsymbol{F}_{m}^{\nu}$-module $W$ satisfies the condition (\#).

It is verified that the above $\left[W_{r, s}\right]$ defines actually the symmetric domain of type (IV) ${ }_{\nu+2},(\mathrm{II})_{5}$ and (EIII) respectively.

The set $\mathfrak{M}_{s}(m, \nu)$ for each $(m, \nu)$ is given as follows.

Case $m=1 . \quad \nu=1, F_{m}^{\nu}=R$.

$$
\mathfrak{M}_{s}(m, \nu)=\left\{\left[W_{r} ; r \in Z, r \geqq 0\right\}, \quad \operatorname{dim} W_{r}=r .\right.
$$

$\left[W_{r}\right]$ defines the symmetric domain of type $(\mathrm{I})_{1,1+r}$.

Case $m=2$.

(a) $\nu=1 . \quad \boldsymbol{F}_{m}^{\nu}=C_{0}=\boldsymbol{R}$.

$$
\mathfrak{M}_{s}(m, \nu)=\left\{\left[W_{0}\right]\right\}, \quad \operatorname{dim} W_{0}=0 .
$$

$\left[W_{0}\right]$ defines the symmetric domain of type (III) ${ }_{2}$.

(b) $\nu=2$. $\boldsymbol{F}_{m}^{\nu}=C_{1}=C$.

$$
\mathfrak{M}_{s}(m, \nu)=\left\{\left[W_{r, 0}\right] ; r \in Z, r \geqq 0\right\}, \quad \operatorname{dim} W_{r, 0}=r .
$$

$\left[W_{r, 0}\right]$ defines the symmetric domain of type $(\mathrm{I})_{2,2+r}$.

(c) $\nu=2 \ell+1(\ell \geqq 1) . \quad F_{m}^{\nu}=C_{2 \ell}$.

$$
\mathfrak{M}_{s}(m, \nu)=\left\{\left[W_{0}\right]\right\}, \quad \operatorname{dim} W_{0}=0 .
$$

$\left[W_{0}\right]$ defines the symmetric domain of type $(\mathrm{IV})_{\nu+2}$.

(d) $\nu=2 \ell+2(\ell \geqq 1) . \quad F_{m}^{\nu}=C_{2 \ell+1}$. 


$$
\nu \neq 4,6 \mathfrak{M}_{s}(m, \nu)=\left\{\left[W_{0,0}\right]\right\}, \quad \operatorname{dim} W_{0,0}=0 .
$$

$\left[W_{0,0}\right]$ defines the symmetric domain of type (IV) ${ }_{\nu+2}$.

$$
\nu=4 \mathfrak{M}_{s}(m, \nu)=\left\{\left[W_{0,0}\right],\left[W_{1,0}\right]\right\}, \quad \operatorname{dim} W_{0,0}=0, \quad \operatorname{dim} W_{1,0}=2 .
$$

$\left[W_{0,0}\right],\left[W_{1,0}\right]$ define symmetric domains of type $(\mathrm{IV})_{6}$, (II $)_{5}$ respectively.

$$
\nu=6 \mathfrak{M}_{s}(m, \nu)=\left\{\left[W_{0,0}\right],\left[W_{1,0}\right]\right\}, \quad \operatorname{dim} W_{0,0}=0, \quad \operatorname{dim} W_{1,0}=4 .
$$

$\left[W_{0,0}\right],\left[W_{1,0}\right]$ define symmetric domains of type (IV) $)_{8}$, (EIII) respectively. Case $m \geqq 3$.

(a) $\nu=1 . \quad F_{m}^{\nu}=R$.

$$
\mathfrak{M}_{s}(m, \nu)=\left\{\left[W_{0}\right]\right\}, \quad \operatorname{dim} W_{0}=0 .
$$

$\left[W_{0}\right]$ defines the symmetric domain of type (III) $)_{m}$.

(b) $\nu=2 . \quad F_{m}^{\nu}=C$.

$$
\mathfrak{M}_{s}(m, \nu)=\left\{\left[W_{r, 0}\right] ; r \in Z, r \geqq 0\right\}, \quad \operatorname{dim} W_{r, 0}=r .
$$

$\left[W_{r, 0}\right]$ defines the symmetric domain of type $(\mathrm{I})_{m, m+r}$.

(c) $\nu=4 . \quad F_{m}^{\nu}=H$.

$$
\mathfrak{M}_{s}(m, \nu)=\left\{\left[W_{0}\right],\left[W_{1}\right]\right\}, \quad \operatorname{dim} W_{0}=0, \quad \operatorname{dim} W_{1}=2 .
$$

$\left[W_{0}\right],\left[W_{1}\right]$ define symmetric domains of type (II $)_{2 m}$, (II $)_{2 m+1}$ respectively. (d) $\nu=8 . \quad F_{m}^{\nu}=K$.

$$
\mathfrak{M}_{s}(m, \nu)=\left\{\left[W_{0}\right]\right\}, \quad \operatorname{dim} W_{0}=0 .
$$

$\left[W_{0}\right]$ defines the symmetric domain of type (EVII).

\section{REFERENCES}

[1] S. Kaneyuki, On the automorphism groups of homogeneous bounded domains, J. Fac. Sci. Univ. Tokyo 14 (1967), 89-130.

[2] W. Kaup, Y. Matsushima and T. Ochiai, On automorphisms and equivalences of generalized Siegel domains, Amer. J. Math. 92 (1970), 475-497.

[ 3 ] K. Nakajima, Some studies on Siegel domains, to appear.

[4] H. Ozeki and M. Takeuchi, On some types of isoparametric hypersurfaces in spheres I, to appear.

[5] I. I. Pjateckii-Šapiro, Géométrie des domaines classiques et théorie des fonctions automorphes, Dunod, Paris, 1966.

[6] I. Satake, Infinitesimal automorphisms of symmetric Siegel domains, to appear.

[7] M. Takeuchi, Homogeneous Siegel domains, Pub. Study Group Geometry, No. 7, Tokyo, 1973.

[8] T. Tsuji, Siegel domains over self-dual cones and their automorphisms, to appear.

[ 9 ] E. B. Vinberg, Homogeneous cones, Soviet Math. Dokl. 1 (1960), 787-790. 
[10] - The theory of convex homogeneous cones, Trans. Moscow Math. Soc. (1963), $340-403$.

[11] — The structure of the group of automorphisms of a homogeneous convex cone, Trans. Moscow Math. Soc. (1965), 63-93.

[12] — S. G. Gindikin and I. I. Pjateckii-Šapiro, Classification and canonical realization of complex bounded homogeneous domains, Trans. Moscow Math. Soc. (1963), 404-437.

Department of Mathematics

Osaka University 